\title{
SU(2) chiral perturbation theory low-energy constants from $2+1$ flavor staggered lattice simulations
}

\author{
Szabolcs Borsányi, ${ }^{1}$ Stephan Dürr, ${ }^{1,2, *}$ Zoltán Fodor, ${ }^{1,2,3}$ Stefan Krieg, ${ }^{1,2}$ \\ Andreas Schäfer, ${ }^{4}$ Enno E. Scholz, ${ }^{4}+{ }_{\dagger}$ and Kálmán K. Szabó ${ }^{1}$ \\ ${ }^{1}$ Bergische Universität Wuppertal, Gaußstr. 20, D-42119 Wuppertal, Germany \\ ${ }^{2}$ Jülich Supercomputing Centre, Forschungszentrum Jülich, D-52425 Jülich, Germany \\ ${ }^{3}$ Institute for Theoretical Physics, Eötvös University, H-1117 Budapest, Hungary \\ ${ }^{4}$ Universität Regensburg, Universitätsstr. 31, D-93053 Regensburg, Germany
}

(Dated: May 4, 2012 and July 24, 2013)

\begin{abstract}
We extract the next-to-leading-order low-energy constants $\bar{\ell}_{3}$ and $\bar{\ell}_{4}$ of SU(2) chiral perturbation theory, based on precise lattice data for the pion mass and decay constant on ensembles generated by the Wuppertal-Budapest Collaboration for QCD thermodynamics. These ensembles feature $2+1$ flavors of two-fold stout-smeared dynamical staggered fermions combined with Symanzik glue, with pion masses varying from 135 to $435 \mathrm{MeV}$, lattice scales between 0.7 and $2.0 \mathrm{GeV}$, while $m_{s}$ is kept fixed at its physical value. Moderate taste splittings and the scale being set through the pion decay constant allow us to restrict ourselves to the taste pseudoscalar state and to use formulas from continuum chiral perturbation theory. Finally, by dropping the data points near $135 \mathrm{MeV}$ from the fits, we can explore the range of pion masses that is needed in $\mathrm{SU}(2)$ chiral perturbation theory to reliably extrapolate to the physical point.
\end{abstract}

PACS numbers: 11.15.Ha, 11.30.Rd, 12.38.Gc 12.39.Fe

\footnotetext{
* E-mail: durr (AT) itp.unibe.ch

$\dagger^{\dagger}$ E-mail: enno.scholz (AT) physik.uni-regensburg.de
} 


\section{INTRODUCTION}

One of the most fascinating aspects of QCD [1], the theory of strong interactions, is the nonanalytic behavior of its Green's functions close to the chiral limit, that is with two or three quark masses taken small, $m_{q} \ll \Lambda^{2} / B$, where $\Lambda \sim 1 \mathrm{GeV}$ is a typical hadronic scale and $B$ is a condensate parameter which we will define (and determine) below.

The structure of these nonanalytic contributions can be worked out in the effective field theory approach which is known as chiral perturbation theory (ChPT) [2, 3]. In this setup physical quantities are expanded in powers of $p / \Lambda$, where $p$ is an external momentum, and the quark mass is treated as $m_{q} \simeq O\left(p^{2}\right)$. Depending on whether this is done for the two $(u, d)$ or three $(u, d, s)$ lightest flavors, the framework is known as $\mathrm{SU}(2)$ or $\mathrm{SU}(3)$ ChPT.

In either case, at the leading order (LO) of the chiral expansion there are two low-energy constants, defined from the pion decay constant and the scalar condensate as

$$
F=\lim _{m_{u}, m_{d} \rightarrow 0} F_{\pi}, \quad B=\lim _{m_{u}, m_{d} \rightarrow 0}\left\{-\langle 0|\bar{q} q| 0\rangle / F_{\pi}^{2}\right\}
$$

where $q$ denotes one of the light flavors (i.e. $q=u$ or $q=d$ ) in the $\mathrm{SU}(2)$ case, and similarly (with also $m_{s}$ sent to 0 ) in the $\mathrm{SU}(3)$ case. At the next-to-leading order (NLO), i.e. at $O\left(p^{4}\right)$ in the chiral counting, seven new low-energy constants show up in the $\mathrm{SU}(2)$ framework [2], or ten low-energy plus two high-energy constants for SU(3) [3]. These low-energy constants parametrize the above-mentioned chiral logarithms in the Green's functions of QCD. Their numerical values can be determined either from experiment or from an ab initio solution of QCD in the relevant (small coupling and light quark mass) regime, as is provided by lattice

QCD [4 6]. Since the chiral logarithms show up as rather subtle effects, meaningful results can only be obtained from lattice data which have excellent statistical precision and explore, at the same time, a wide enough range of lattice spacings, quark masses, and box volumes such that all sources of systematic error can be controlled and eventually removed.

In this paper we provide such a determination of the $\mathrm{SU}(2)$ low-energy constants $\bar{\ell}_{3}$ and $\bar{\ell}_{4}$. Their numerical values are extracted from the quark-mass dependence of $M_{\pi}$ and $F_{\pi}$, respectively, and complemented by numerical values of the leading-order low-energy constants $F$ and $B$. We use staggered fermion simulations with $N_{f}=2+1$ dynamical flavors, that is, two degenerate light quarks of variable mass $m_{l}$ and an active strange quark whose mass $m_{s}$ is pinned down at its physical value. As a result of this our values of the lowenergy constants are supposed to coincide with those in the real word. The inverse lattice 
spacings cover the range $0.7 \mathrm{GeV} \leq a^{-1} \leq 2.0 \mathrm{GeV}$ (see Sec.【II and Table If for details). A preliminary account of our work (based on a smaller dataset) was given in Ref. [7].

The remainder of this paper is structured as follows. In Sec.[I] we specify the gauge and fermion actions used and list the ensembles which go into the determination of the chiral low-energy constants. Furthermore, details are given how we calculate the pion mass and decay constant, and how we correct the latter for the effect of the finite spatial volume of the box (which is always a small correction, since our data satisfy $3.3 \leq M_{\pi} L \leq 6.8$ ). In Sec.III we specify the procedure through which we set, for each bare coupling $\beta=6 / g_{0}^{2}$, the lattice spacing $a$ and the physical values of the bare quark masses $m_{l}=\left(m_{u}+m_{d}\right) / 2$ and $m_{s}$. Section IV contains the core part of the present investigation, an analysis of our data with $\mathrm{SU}(2) \mathrm{ChPT}$ at NLO, with details of how we select adequate mass windows and determine the systematic uncertainty of the fitted low-energy constants. Section $\nabla$ contains a similar though less mature analysis at next-to-next-to-leading order (NNLO), where again the main goal is to determine the NLO coefficients, with and without the help of some priors on the remaining NLO and NNLO low-energy constants. This helps to give a reliable estimate of the theoretical uncertainty of the NLO results obtained. A summary and a comparison with the findings of other recent lattice studies of SU(2) NLO low-energy constants is presented in Sec.VI.

\section{LATTICE DATA}

In this section we specify the lattice actions used, list the ensembles which go into the determination of the $\mathrm{SU}(2)$ low-energy constants, give details of how we extract the pion mass and decay constant on a given ensemble, and describe the procedure by which we remove the (small) impact of the finite spatial box size $L$ on the data.

\section{A. Lattice action and ensemble generation}

The lattices are generated with a tree-level Symanzik improved gauge action [8] and 2+1 flavors of staggered quarks with two levels of stout-smearing [9]. The action is specified in full detail in Ref. [10]. The algorithm used is a combination of HMC and RHMC with standard improvements (see, e.g., Ref. [11] for an overview). Some ensembles were generated for scale 
setting purposes in previous finite temperature studies [10, 12 16], and some were generated specifically for the present investigation. The taste splitting $M_{P X}^{2}-M_{P P}^{2}$ [where the first subscript indicates that the state is a pseudoscalar in spinor space and the second one refers to its taste, with PP indicating the Goldstone state that couples to the operator $\left.\left(\gamma_{5} \otimes \xi_{5}\right)\right]$ is in good approximation independent of the quark mass [17]. Building on this information, the masses of the taste partners occurring in the present investigation can be reconstructed from the Goldstone masses given below and the splittings presented in Refs. [15, 16].

We adopt a mass independent scale setting, that is the lattice spacing $a$ depends only on the coupling $\beta$ in the gauge action, not on the quark masses $m_{l}, m_{s}$. With this choice it is straightforward to adjust, for each $\beta$, the strange quark mass roughly to its physical value by tuning the ratio $\left(2 M_{K}^{2}-M_{\pi}^{2}\right) / M_{\phi}^{2}$ to its physical value. In the numerator the FLAG values [18] of the pseudoscalar meson masses are used which correct for isospin breaking and QED effects (cf. Sec.III below). In the denominator the PDG value [19] of the vector meson mass is used, despite the fact that our $M_{\phi}$ involves only the connected contribution (the difference is believed to be small, cf. Ref. [11]). Starting from the symmetric point $m_{l}=m_{s} \simeq m_{s}^{\text {phys }}$, one can lower the light quark mass $m_{l}$, at fixed $m_{s}$, until the ratio $M_{\pi}^{2} / f_{\pi}^{2}$ assumes its physical value. This is one possible definition of the physical point in which we effectively set the lattice spacing through $f_{\pi}$ with details given in Sec.III (other definitions differ from this one just in the choice of which ratios are affected by cutoff effects and which are not).

From a more practical point of view it suffices to say that we simulate, for each $\beta$, a number of $\left(m_{l}, m_{s}\right)$ combinations, where $m_{s}$ is held fixed and is close to whichever definition of the physical strange quark mass that one may adopt, while $m_{l}$ varies between roughly the physical light quark mass and four to ten times this value (depending on $\beta$ ). The

precise value of $m_{l}^{\text {phys }}$ is determined, a posteriori, by means of an interpolation, as described in Section III below. A summary of our ensembles and their bare parameters is given in Table I.

\section{B. Calculating meson masses and decay constants}

A specific advantage of staggered fermions (or of any other discretization with some form of chiral symmetry) is that the decay constant $f=\sqrt{2} F$ of a pseudoscalar meson (in the 


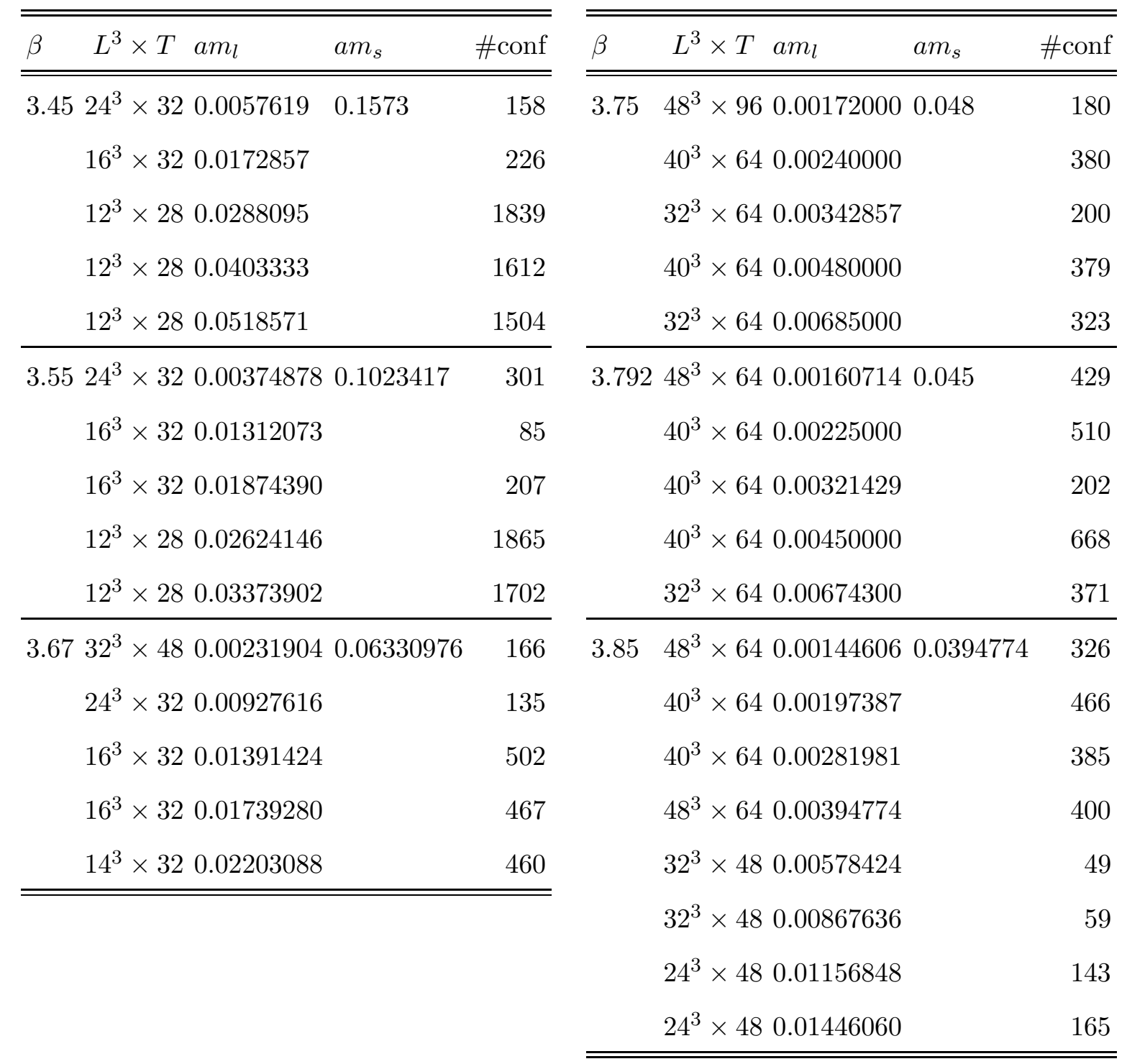

TABLE I. Overview of the staggered $2+1$ flavor ensembles used in this work.

following we will distinguish the two normalizations by using either the upper-case or the lower-case symbol) to the zero component of an axial current can be extracted without recurrence to any lattice-to-continuum matching factor.

We start from the two-point function $C_{P P}(t)$ between two pointlike pseudoscalar density operators (at least one of which is projected to zero spatial momentum) which, for an intermediate window of the Euclidean time $t$, takes the form

$$
C_{P P}(t)=A_{P P}[\exp (-M t)+\exp (-M(T-t))]
$$

with $T$ the lattice extent in the fourth direction. The mass $M$ corresponds to the mass of the lightest asymptotic state with the right quantum numbers (here $\pi$ or $K$ ), whereas the 
amplitude $A_{P P}$ is proportional to the squared matrix element, i.e. $A_{P P} \propto\langle 0|P| x y\rangle^{2} / M$, where $|x y\rangle$ denotes the pseudoscalar state put together from flavors $x, y$ (here $x, y=l, s$ ).

In practice it means that we determine, in a first step, the mass and the amplitude from the PP correlator. We do this either via the effective mass and amplitude method where the determination of

$$
\begin{aligned}
M_{\mathrm{eff}}(t) & =\frac{1}{2} \log \left(\frac{C(t-1)+\sqrt{C(t-1)^{2}-C(T / 2)^{2}}}{C(t+1)+\sqrt{C(t+1)^{2}-C(T / 2)^{2}}}\right) \\
A_{\mathrm{eff}}(t) & =\frac{C(t)}{\exp \left[-M_{\mathrm{eff}}(t) t\right]+\exp \left[-M_{\mathrm{eff}}(t)(T-t)\right]}
\end{aligned}
$$

is followed by a fit to a constant over some time region $t \in\left[t_{\min }: t_{\max }\right]$, or using a direct fit of the correlator to the functional form (2). In either case the data are symmetrized about $T / 2$, and $t_{\max } \leq T / 2$. The decay constant is defined as $f_{x y}=\left\langle 0\left|A_{4}\right| x y\right\rangle / M_{x y}$, and via the $\mathrm{PCAC}$ relation this is transformed into

$$
f_{x y}=\left(m_{x}+m_{y}\right) \frac{\langle 0|P| x y\rangle}{\sqrt{2} M_{x y}^{2}}
$$

where $m_{x, y}$ denotes the quark mass of the flavor $x$ or $y$. Putting things together, it follows that the decay constant may be obtained from the amplitude and the mass as

$$
f_{x y} \propto\left(m_{x}+m_{y}\right) \sqrt{\frac{A_{x y}}{M_{x y}^{3}}}
$$

where the missing prefactors (e.g. $L^{3}$ ) reflect normalization conventions which depend on the geometry, but not on the quark masses.

For the interim step, i.e. the determination of $M_{\text {eff }}, A_{\text {eff }}$ from the correlators, a typical plateau is shown in Fig.1. We looked for a potential zig-zag of the data close to the mid-time point $T / 2$. This, if present, is commonly attributed to a back-propagating parity partner [17] and reflects an effect which is specific to the staggered discretization. An advantage of the symmetric definition of the effective mass (3) is that $M_{\text {eff }}(t)$ for an odd value of $t$ uses only data from the original correlator at even $t$ and vice versa. Accordingly, we can compare the results of $(i)$ a plateau average of $M_{\text {eff }}(t)$ for odd $t,(i i)$ a plateau average of $M_{\text {eff }}(t)$ for even $t$, and (iii) the result of a direct fit to the Ansatz (2) (which does not distinguish between even and odd time-slices). We have carefully analyzed the impact of these options and found them completely insignificant compared to both the statistical uncertainty and (even more so) the theoretical uncertainty inherent in the precise choice of the masses and 

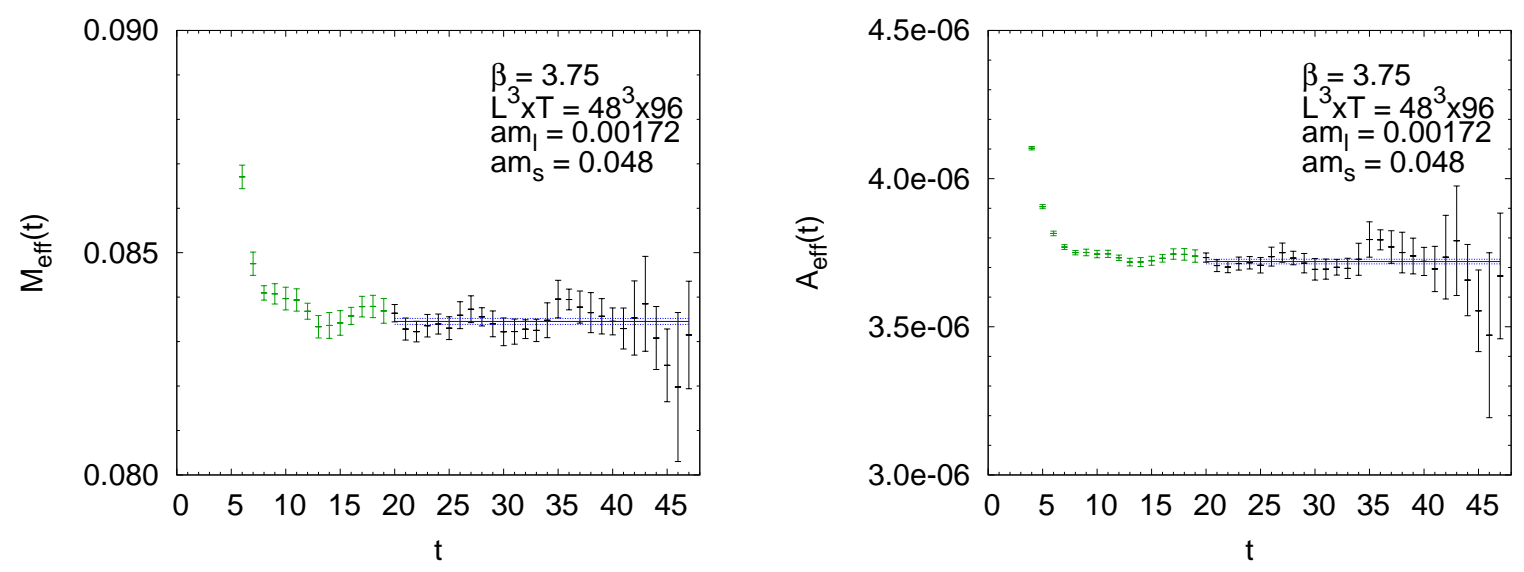

FIG. 1. Effective masses (left panel) and amplitudes (right panel) from the pion correlator at $\beta=3.75, a m_{l}=0.00172, a m_{s}=0.048$, including the plateau-fits (blue lines).

lattice spacings included in the chiral fit. The latter represent relevant options that will be discussed in detail in Sections IV and $\mathrm{V}$ below.

The statistical errors are determined via a jackknife procedure (using an extension known as superjackknife [20, 21] which allows one to deal with ensembles of unequal size). Typically the data are blocked in sets of 1,2,5,10 configurations [where a configuration corresponds to $O(10)$ trajectories], and we determine at which level the jackknife error saturates.

We stress that all the fitting is performed within the jackknife procedure. A common issue in many lattice calculations is that the covariance matrix (in Euclidean time direction) of the local masses - e.g. the $28 \times 28$ matrix $C$ that corresponds to $t \in[20, \ldots, 47]$ of Fig. [1 - may not be invertible, at least not on all jackknife samples. This precludes a clean-cut definition of the $\chi^{2}$ of such a fit to the primary data; one often truncates $C$ to its diagonal and neardiagonal parts or uses pseudoinverses based on the singular value decomposition of $C$ to come up with a modified $\chi^{2}$. We find that the "fit within jackknife" approach yields very robust values of the statistical error of the fitted mass plateaus, regardless of which effective $C^{-1}$ is used. In the end we opted for using uncorrelated fits to the primary data to avoid an uninvertible correlation matrix in some occasions. The way in which the correlations among the secondary data $a^{-1}, M_{x y}, f_{x y}$ are treated will be discussed in Sec.IVA below. 


\section{Finite-volume corrections}

Pseudoscalar masses and decay constants experience a systematic shift due to the finite spatial box length $L$. Approximate three-loop and two-loop expressions have been given for the ratios $M_{\pi}(L) / M_{\pi}(\infty)$ and $f_{\pi}(L) / f_{\pi}(\infty)$, respectively, in Ref. [22]. For the range of quark masses used in this work they are supposed to give a reliable estimate of these (small) shifts. The numerical values thus obtained vary between 0.1 and 2.7 per mil for the pion mass, and between 0.2 and 7.5 per mil for the pion decay constant. For each ensemble we thus calculate these ratios and apply them to our data. In the following, only the finite-volume corrected data are used.

\section{Other systematic uncertainties}

There is a number of systematic uncertainties which we cannot estimate from our data set, since they are not systematically probed or varied. These include the effect that a slight mistuning of the dynamical strange quark mass has on the chiral low-energy constants, the effect of the quenching of the charm quark, and the way in which we correct for the fact that isospin is broken (by both electromagnetism and $m_{u} \neq m_{d}$ ) in nature but not in our simulations. The size of such effects can only be assessed by means of theoretical arguments; see, e.g., the discussion in the FLAG report [18]. For instance a slight mistuning of the dynamical strange quark mass can be believed to be tiny, since the FLAG compilation could not even detect a statistically significant difference between $\mathrm{SU}(2)$ low-energy constants determined from $N_{f}=2$ and $N_{f}=2+1$ simulations. In consequence a similar statement

holds with respect to the quenching of the charm quark. Estimates of the impact of isospin breakings on the definition of the physical values of $M_{\pi}$ and $f_{\pi}$ are found in Ref. [18]. We find that all theoretically accounted sources of systematic uncertainty are negligible compared to the systematic uncertainties that emerge from the chiral fits (see Secs. [V] and $\mathrm{V}]$ below).

\section{DETERMINING THE LATTICE SCALE AND PHYSICAL QUARK MASS}

As indicated in the previous section, we wish to determine for each $\beta$ the lattice spacing $a$ and the physical value of $a m_{l}$. Since the simulation points for any $\beta$ are at a fixed value of $a m_{s}$ (which is tuned to its physical value, see Sec.IIA), it is clear that the observables 
to be used shall include only the light but not the strange flavor, and the obvious choice is thus $f_{\pi}=\sqrt{2} F_{\pi}$ and $M_{\pi}$.

On a more technical level we proceed by means of a two-step procedure. First, we extrapolate the ratio $\left(a M_{l l}\right)^{2} /\left(a f_{l l}\right)^{2}=\left(a M_{\pi}\right)^{2} /\left(a f_{\pi}\right)^{2}$ of the squared pion mass and decay constant to its physical value, 1.06846. Here we use the isospin averaged and electromagnetically corrected pion mass $M_{\pi}^{\text {phys }}=134.8 \mathrm{MeV}$ from FLAG [18], and the PDG value of the pion decay constant $f_{\pi}^{\text {phys }}=130.41 \mathrm{MeV}$ [19]. In this step the purpose of the square is to reduce the amount of curvature, and we interpolate the data by means of a low-order polynomial and rational Ansatz (typically with three parameters applied to the five lightest data points, i.e., with two degrees of freedom). We stress that the point where this ratio assumes the desired value, 1.06846 , is always very close to the lightest simulated quark mass. In view of this it should not come as a surprise that the values of $a m_{l}^{\text {phys }}$ that stem from the polynomial and the rational fit are always very close to each other (on the scale set by the statistical error). We use the average of the two as our central value; the difference should be seen as indicative of the systematic uncertainty of $a m_{l}^{\text {phys }}$ from this set of ensembles.

In the second step we consider $a f_{\pi}$ as a function of $a m_{l}$. Again, we interpolate the data with the same polynomial and rational Ansatz, and determine the ordinate value at the abscissa point that was specified in the previous step. This value $a f_{\pi}$ is then identified with the product of the lattice spacing $a$ and the PDG value $f_{\pi}^{\text {phys }}=130.41 \mathrm{MeV}[19]$; this yields the lattice spacing $a$ in $\mathrm{fm}$ for the lattice theory at that particular value of the coupling $\beta$. A typical example of this two-step procedure is shown in Fig.2 in the relevant range (close to the lightest mass point) the difference between the two Ansätze is invisibly small. Furthermore, in Table \the results for the physical light quark masses and lattice spacings obtained by this method are displayed.

As a final comment, let us remark that already in these two steps one could, in principle, use ChPT. We rather prefer to stay with the simple yet robust procedure as sketched above. This ensures that the fact that some chiral fits go wild (when an inadequate fitting window is used, cf. the discussion in Secs. IV and $(\nabla)$ is not linked to a potential mishap in the physical mass and scale determination. In other words, we take the lattice spacing and the physical light quark mass from an "ideal" simulation where $M_{\pi} / f_{\pi}$ is exactly tuned to its physical value, and use this knowledge regardless of how many data points enter the chiral fits described below. Needless to comment that for those NLO and NNLO fits which 

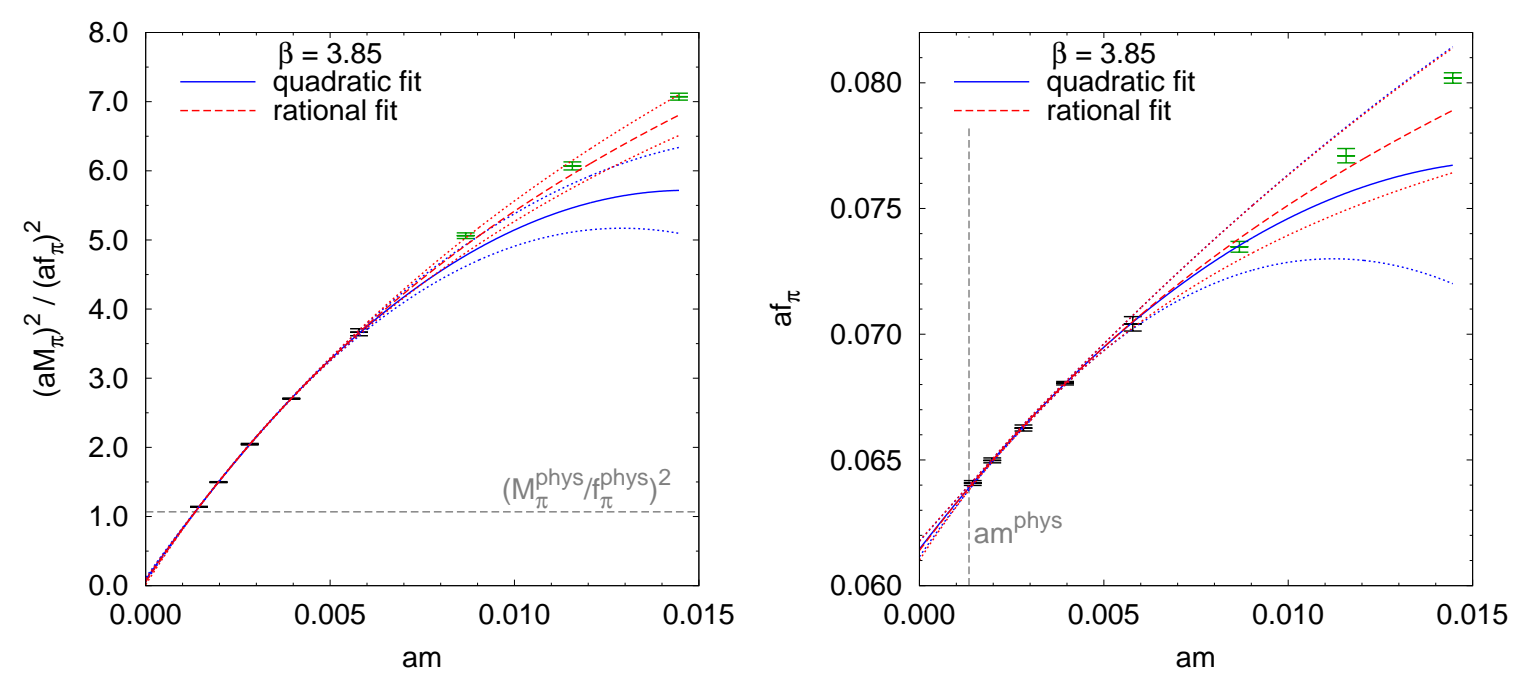

FIG. 2. Fits to the ratio $\left(a M_{\pi}\right)^{2} /\left(a f_{\pi}\right)^{2}$ (left) and the decay constant $a f_{\pi}$ (right) at $\beta=3.85$ to obtain the physical light quark mass $a m^{\text {phys }}$ and the lattice scale $1 / a$. Shown are quadratic (solid blue lines) and rational (dashed red lines) fits which include the black data points; the green data points were excluded from the fits. Error bands are indicated by dotted lines. The dashed gray lines mark the physical value of the ratio and the physical light quark mass extracted therefrom.

\begin{tabular}{cccc}
\hline \hline$\beta$ & $a m^{\text {phys }}$ & $1 / a / \mathrm{GeV}$ & $a / \mathrm{fm}$ \\
\hline 3.45 & $5.771(18) \cdot 10^{-3}$ & $0.69468(67)$ & $0.28406(27)$ \\
3.55 & $3.612(18) \cdot 10^{-3}$ & $0.9165(12)$ & $0.21531(28)$ \\
3.67 & $2.191(15) \cdot 10^{-3}$ & $1.3063(25)$ & $0.15105(29)$ \\
3.75 & $1.6889(55) \cdot 10^{-3}$ & $1.6288(15)$ & $0.12115(11)$ \\
3.792 & $1.5355(70) \cdot 10^{-3}$ & $1.7935(22)$ & $0.11002(14)$ \\
3.85 & $1.3430(98) \cdot 10^{-3}$ & $2.0410(35)$ & $0.09668(16)$ \\
\hline \hline
\end{tabular}

TABLE II. Physical light quark mass $a m^{\text {phys }}$, lattice scale $1 / a$ and spacing $a$ at different $\beta$ obtained by the method described in Sec. III.

work fine (and which include the lightest data points), their intrinsic physical mass and scale determination were always found to be in very good agreement with the result of the procedure described in the previous two paragraphs. 


\section{FITS TO NLO-SU(2) CHPT}

In this section we will describe how we fit the quark-mass dependence of the meson decay constant and its squared mass to the prediction of NLO ChPT and in this way obtain the low-energy constants (LECs) appearing in these ChPT formulas. Since in the simulations considered here the strange quark mass was fixed to its physical value, we will only deal with the light quark-mass dependence described by SU(2) ChPT. The LECs extracted in this way therefore contain the correct contribution of the effects due to the strange quark present in nature. Further, we are restricting ourselves to the case of a degenerate light quark mass, which we will denote by $m$ (or am in lattice units) throughout the remainder of this paper, i.e., from now on we drop the subscript $l$ on the light quark-mass parameter. At the physical point, this mass corresponds to the average mass of the two light quarks observed in nature: $m^{\text {phys }}=\left(m_{u}^{\text {phys }}+m_{d}^{\text {phys }}\right) / 2$.

In the following we will try to fit our data to continuum ChPT and not consider variants of ChPT, which take into account lattice discretization effects and/or taste violations present in the staggered formulation; see, e.g., Refs. [17, 23, 24]. Whether a continuum Ansatz is suitable to describe our data is not a priori clear and needs to be tested. It is valid if, within the statistical precision of our data, no cutoff dependence is seen in the observables considered. As will be shown below, this is indeed the case for our $M_{\pi}$ and $f_{\pi}$ data in the relevant region. This can be traced back to the combined effect of (a) the two levels of stout smearing in the action (see Sec.III) and (b) the specific choice of our scaling trajectory, i.e., the scale and the physical quark mass being set through $f_{\pi}$ and $M_{\pi}$ (see Sec.III). While (b) ensures that discretization effects on both $M_{\pi}$ and $f_{\pi}$ vanish at the physical point, (a) keeps them small in its vicinity due to the suppression of taste violations (see, e.g., Refs. [25, 26]

and [15]). As we will see below, the combination of these two effects leads to a suppression of discretization effects within our statistical precision over a sufficiently wide region of quark masses and lattice spacings. 


\section{A. Methods and fit formulas}

ChPT up to NLO predicts for the decay constant $f_{\pi}$ and the squared mass $M_{\pi}^{2}$ of a meson consisting of two mass-degenerate quarks of mass $m$ the following functional form [2, 3, 18]

$$
\begin{aligned}
M_{\pi}^{2} & =\chi\left[1+\frac{\chi}{16 \pi^{2} f^{2}} \log \frac{\chi}{\Lambda_{3}^{2}}\right], \\
f_{\pi} & =f\left[1-\frac{\chi}{8 \pi^{2} f^{2}} \log \frac{\chi}{\Lambda_{4}^{2}}\right], \\
\chi & =2 B m .
\end{aligned}
$$

At this order four LECs appear: the decay constant $f$ in the $\mathrm{SU}(2)$-chiral limit $(m \rightarrow 0)$, the condensate parameter $B$, and two low-energy scales $\Lambda_{3}$ and $\Lambda_{4}$. The condensate parameter $B$ depends like the quark mass $m$ on the renormalization scheme, but the combination $\chi$ is renormalization scheme independent, and it is this combination which will be used exclusively in this work. The low-energy scales are related to the LECs $\bar{\ell}_{3}$ and $\bar{\ell}_{4}$ at the scale of the physical pion mass $M_{\pi}^{\text {phys }}$ via

$$
\bar{\ell}_{i}=\log \frac{\Lambda_{i}^{2}}{\left(M_{\pi}^{\text {phys }}\right)^{2}}, \quad i=3,4 .
$$

Since we used the physical values $f_{\pi}^{\text {phys }}$ and $\left(M_{\pi}^{\text {phys }}\right)^{2}$ to set the scale and determine the physical light quark mass $m^{\text {phys }}$ for each set of lattice ensembles with a given gauge coupling $\beta$, our Ansatz should reproduce the physical point. Note also, that each set of lattice ensembles contained one simulated point in close vicinity of the physical point. Therefore, we could impose the following constraints to the chiral formulas [Eqs. (77) and (8)]):

$$
\left.M_{\pi}^{2}\right|_{m=m^{\text {phys }}}=\left(M_{\pi}^{\text {phys }}\right)^{2},\left.\quad f_{\pi}\right|_{m=m^{\text {phys }}}=f_{\pi}^{\text {phys }} .
$$

As it is easily derived, these two constraints result in the relations

$$
\begin{aligned}
& \log \frac{\chi^{\text {phys }}}{\Lambda_{3}^{2}}=\frac{16 \pi^{2} f^{2}}{\left(\chi^{\text {phys }}\right)^{2}}\left(\left(M_{\pi}^{\text {phys }}\right)^{2}-\chi^{\text {phys }}\right), \\
& \log \frac{\chi^{\text {phys }}}{\Lambda_{4}^{2}}=\frac{8 \pi^{2} f^{2}}{f_{\pi}^{\text {phys }}}\left(f-f_{\pi}^{\text {phys }}\right)
\end{aligned}
$$

between the LECs, where $\chi^{\text {phys }}=2 B m^{\text {phys }}$. Using them to eliminate, e.g., the low-energy scales from the NLO ChPT formulas, the constrained formulas can be written as

$$
\begin{aligned}
M_{\pi}^{2} & =\chi\left[1+\frac{\chi}{16 \pi^{2} f^{2}} \log \frac{\chi}{\chi^{\text {phys }}}+\frac{\chi}{\chi^{\text {phys }}} \frac{\left(M_{\pi}^{\text {phys }}\right)^{2}-\chi^{\text {phys }}}{\chi^{\text {phys }}}\right], \\
f_{\pi} & =f\left[1-\frac{\chi}{8 \pi^{2} f^{2}} \log \frac{\chi}{\chi^{\text {phys }}}-\frac{\chi}{\chi^{\text {phys }}} \frac{f-f_{\pi}^{\text {phys }}}{f}\right] .
\end{aligned}
$$


Note that $\chi / \chi^{\text {phys }}=m / m^{\text {phys }}$. These formulas now depend on two LECs, $B$ and $f$, and the two physical input values $M_{\pi}^{\text {phys }}$ and $f_{\pi}^{\text {phys }}$. In our fitting procedure only $\chi^{\text {phys }}$ and $f$ will be treated as free parameters. For that reason, we also like to refer to these fits as parameter-reduced fits. [Of course, by treating $M_{\pi}^{\text {phys }}$ and $f_{\pi}^{\text {phys }}$ as free parameters as well, one would recover the unconstrained Eqs. (77) and (8), respectively.]

In the following we want to perform combined (i.e., fitting $M_{\pi}^{2}$ and $f_{\pi}$ simultaneously) global fits to our lattice data at the different gauge couplings available to us. For this reason we make use of the lattice scale and physical light quark mass determined beforehand (see Sec.(III). The meson mass and decay constant in lattice units at a given gauge coupling $\beta$ are converted into physical units by

$$
M_{\pi}^{2}=(1 / a)_{\beta}^{2}\left(a M_{\pi}\right)_{\beta}^{2}, \quad f_{\pi}=(1 / a)_{\beta}\left(a f_{\pi}\right)_{\beta},
$$

respectively. Furthermore, we rewrite the combination $\chi=2 B m$ as

$$
\chi=\left(2 B m^{\text {phys }}\right) \frac{(a m)_{\beta}}{\left(a m^{\text {phys }}\right)_{\beta}}=\chi^{\text {phys }} \frac{(a m)_{\beta}}{\left(a m^{\text {phys }}\right)_{\beta}}
$$

and will determine only the renormalization-independent combination $\chi^{\text {phys }}=2 B m^{\text {phys }}$ in our fits. The factor $m^{\text {phys }}$ will be removed, based on external data, in Sec.VI

It follows that with this setup, there is no correlation between secondary quantities (the pion mass $M_{\pi}$ and decay constant $f_{\pi}$ ) from ensembles with different $\beta$ (cf. Table $\mathbb{I}$ ), but there is a substantial correlation among $M_{\pi}$ and $f_{\pi}$ on any individual ensemble (because they are extracted from the same pseudoscalar correlator), and there is a weak correlation among all $M_{\pi}$ and $f_{\pi}$ with the same value of $\beta$ (because the scale setting attributes a joint $(1 / a)_{\beta}$ and $\left(a m^{\text {phys }}\right)_{\beta}$ to all ensembles with a common $\left.\beta\right)$. As explained at the end of Sec.IIB we perform all fitting within the jackknife procedure. In the present context this means that the statistical uncertainty from the scale setting is propagated into the uncertainty of the fitted NLO ChPT low-energy constants. Similarly to what was reported at the end of Sec.IB for primary quantities, we now observe a genuine robustness of the fitted parameters to the details of the pseudoinverse that is formed from the covariance matrix among the secondary observables. The respective correlated $\chi^{2}$ and $p$-values of the fit do, however, show a clear sensitivity to the details of the pseudoinverse. Therefore we decided to always quote results obtained with uncorrelated fits, but with the statistical uncertainty (of both the fitted parameters and the uncorrelated $\chi^{2}$ values) determined through the outer jackknife procedure (using the superjackknife extension described in Refs. [20, 21]). 


\section{B. Combined global fits}

\section{NLO ChPT without constraints}

We begin the discussion of the chiral fits with the results of applying the unconstrained fit formulas, Eqs. (7) and (8), to our data. The combined fit has four free parameters: $\chi^{\text {phys }}=2 B m^{\text {phys }}, f, \Lambda_{3}$, and $\Lambda_{4}$. A priori, it is not clear whether all simulated lattice spacings will lie in the scaling region, especially for the very coarse lattices with lattice spacings of up to $0.28 \mathrm{fm}$ (corresponding to a lattice scale of $1 / a \approx 0.7 \mathrm{GeV}$ ) this is questionable. Also, the range of quark masses or equivalently meson masses to which NLO-SU(2) ChPT is applicable will have to be determined. Eventually, the fit quality, which we measure by the standard $\chi^{2} /$ d.o.f., will be used to decide on these issues. In Fig. [3] we provide a landscape of the simulated meson masses at the various lattice spacings. The horizontal dashed lines indicate the various mass cuts we applied in our global combined fits. One should keep in mind that for the combined fit, each point in the landscape plot represents two data points: one for the meson mass and one for the meson decay constant.

In Fig. [4 we show the result of a combined fit to the meson masses and decay constants to the data at all available lattice spacings and a mass range of $135 \mathrm{MeV} \leq M_{\pi} \leq 390 \mathrm{MeV}$, i.e. excluding only the heaviest point at each simulated $\beta$ value. These plots show all available data points, whether or not included in the fit range. We marked those points which have been excluded by green symbols, while points included in the fit range are marked by black symbols. As one can already judge by eye, this fit gives a bad $\chi^{2} /$ d.o.f. of about 4.1 (with \#d.o.f. $=58-4=54$ ), although the fitted parameters are in a reasonable range, cf. Fig. In a first step, we consider reducing the mass range by excluding more and more of the heavier points as indicated by the dashed horizontal lines in our landscape plot, Fig. 3. In Fig. 5 we show how the fitted parameters and the $\chi^{2} /$ d.o.f. vary when we change the mass range in the fit, still considering the ensembles at all available lattice spacings. First we focus on those fit ranges including the near physical points which are shown above the topmost dashed horizontal line in each of the plots in Fig. 5. (In the fit results shown below that line, the near physical points and subsequently other points with light meson masses have been excluded; we will comment on those results below.) As one can see, the $\chi^{2} /$ d.o.f. improves by narrowing the fit range, and for the range $135 \mathrm{MeV} \leq M_{\pi} \leq 240 \mathrm{MeV}$, it is already 


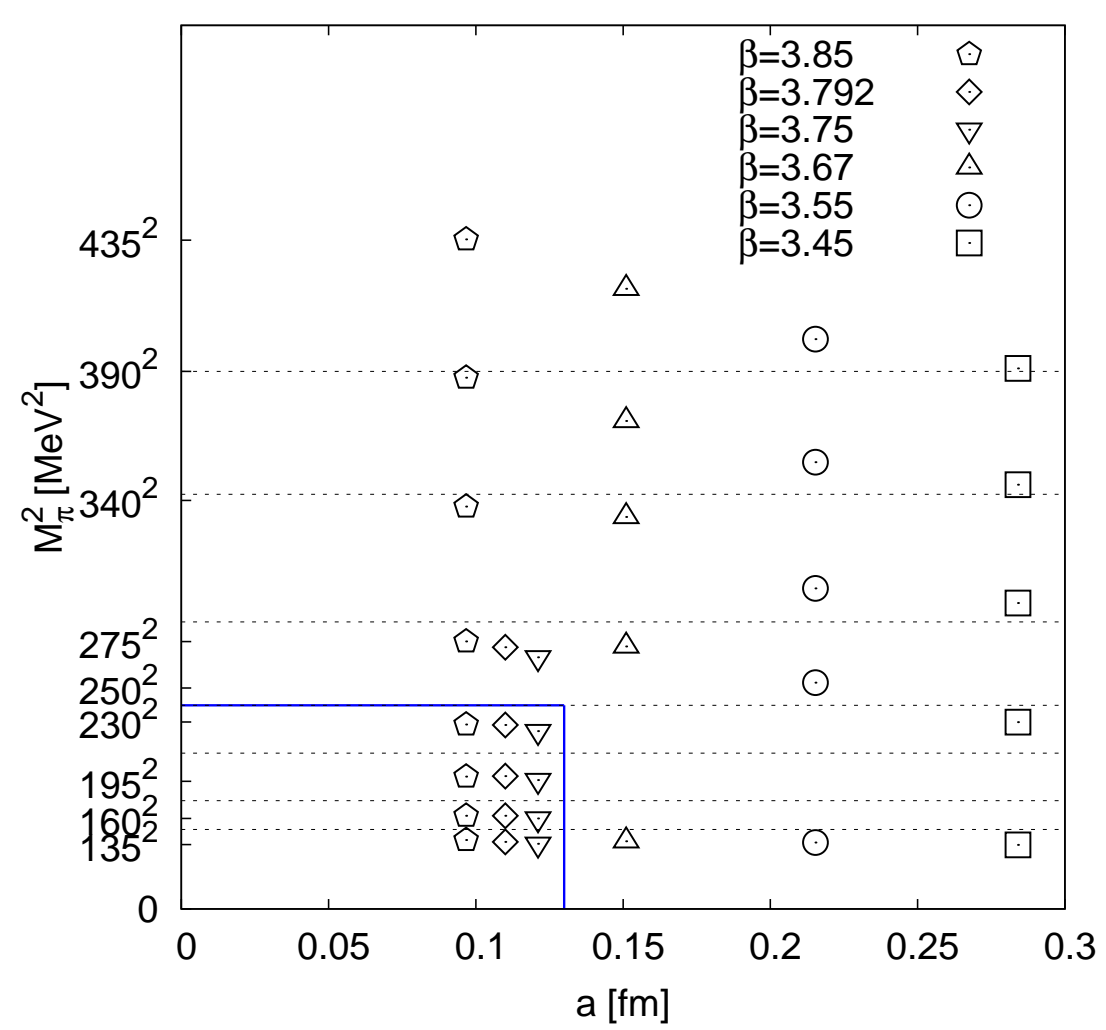

FIG. 3. Landscape plot of the simulated pion masses $M_{\pi}^{2}$ and lattice spacings $a$. The horizontal dashed lines indicate the various mass cuts applied in our chiral fits. The full blue line indicates our preferred fit range $\left(a<0.12 \mathrm{fm}, M_{\pi}<240 \mathrm{MeV}\right)$, see text for details.

comparable with 1.0. The four fit parameters do not plateau yet, but their magnitude seems to settle. Note, that for the two narrowest fit ranges (upper mass limit at 160 or $195 \mathrm{MeV}$ ), the number of available data points might be too small as is also indicated by the larger error bars at these ranges. We already show in these plots the central values and error bands as determined from our preferred fit below, just to demonstrate that these error bands are compatible with the values obtained from fits to all ensembles. For completeness, in Fig.6 we show the LECs $\bar{\ell}_{3}, \bar{\ell}_{4}$ and the phenomenologically relevant ratio $f_{\pi}^{\text {phys }} / f$ as determined from our fits with different mass ranges.

In a second step, we will now examine whether or not all the available lattice spacings already lie in the scaling region. Remember that since the meson mass and decay constant define our scaling trajectory, no terms modelling discretization effects have been added to 

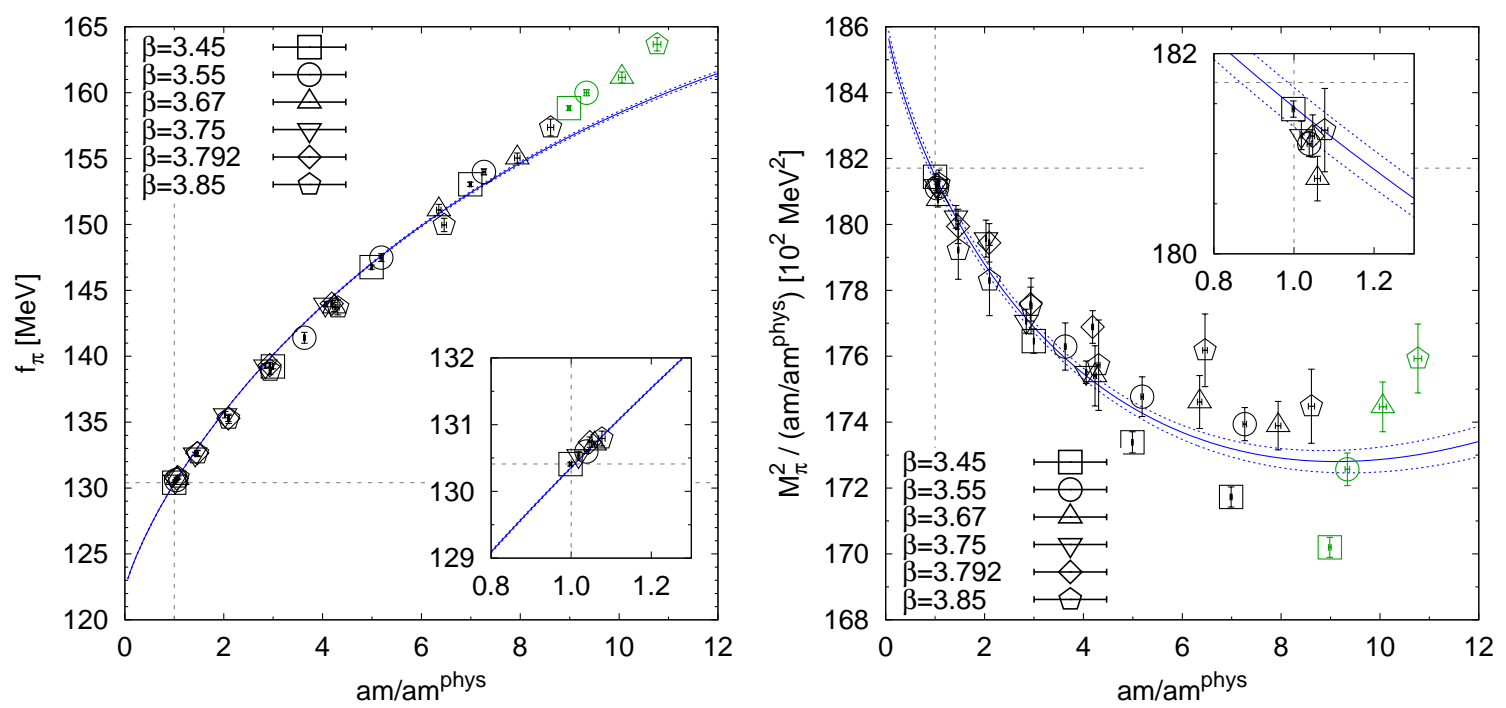

FIG. 4. Combined fit for all lattice scales, $135 \mathrm{MeV} \leq M_{\pi} \leq 390 \mathrm{MeV}$. Left panel: meson decay constant, right panel: squared meson mass divided by quark-mass ratio. Points marked by black symbols are included in the fit, those marked by green symbols are excluded. The physical values are indicated by dashed gray lines.

our chiral formulas. To test the scaling behavior, we excluded ensembles belonging to one or more gauge coupling $\beta$ from the fits. In Fig. 7 we show how the fit parameters and $\chi^{2} /$ d.o.f. change with respect to which lattice spacings are included in the fits. We show this for two different mass ranges, both including the near physical points. The leftmost point on each of these plots is from a fit to all available lattice spacings. Then, separated by vertical dashed lines, groups of fits follow where one, two, three or four lattice spacings have been excluded. The horizontal blue lines show our final estimate with error bands of the quantity displayed. Especially in the group where three lattice spacings have been excluded (the second from the right), we observe the parameters to reach a plateau by excluding the coarse lattice spacings. Overall, it is also reassuring that all the points fall into the error band of the combined statistical and systematic error, which we are going to discuss in the remainder now.

From the previous discussion about excluding coarser lattice spacings from the chiral analysis, we decided to restrict the range in the lattice spacings to $1 / a>1.6 \mathrm{GeV}$ or $a \leq 0.12 \mathrm{fm}$, i.e., only the ensembles at gauge couplings $\beta=3.75,3.792$, and 3.85 will 

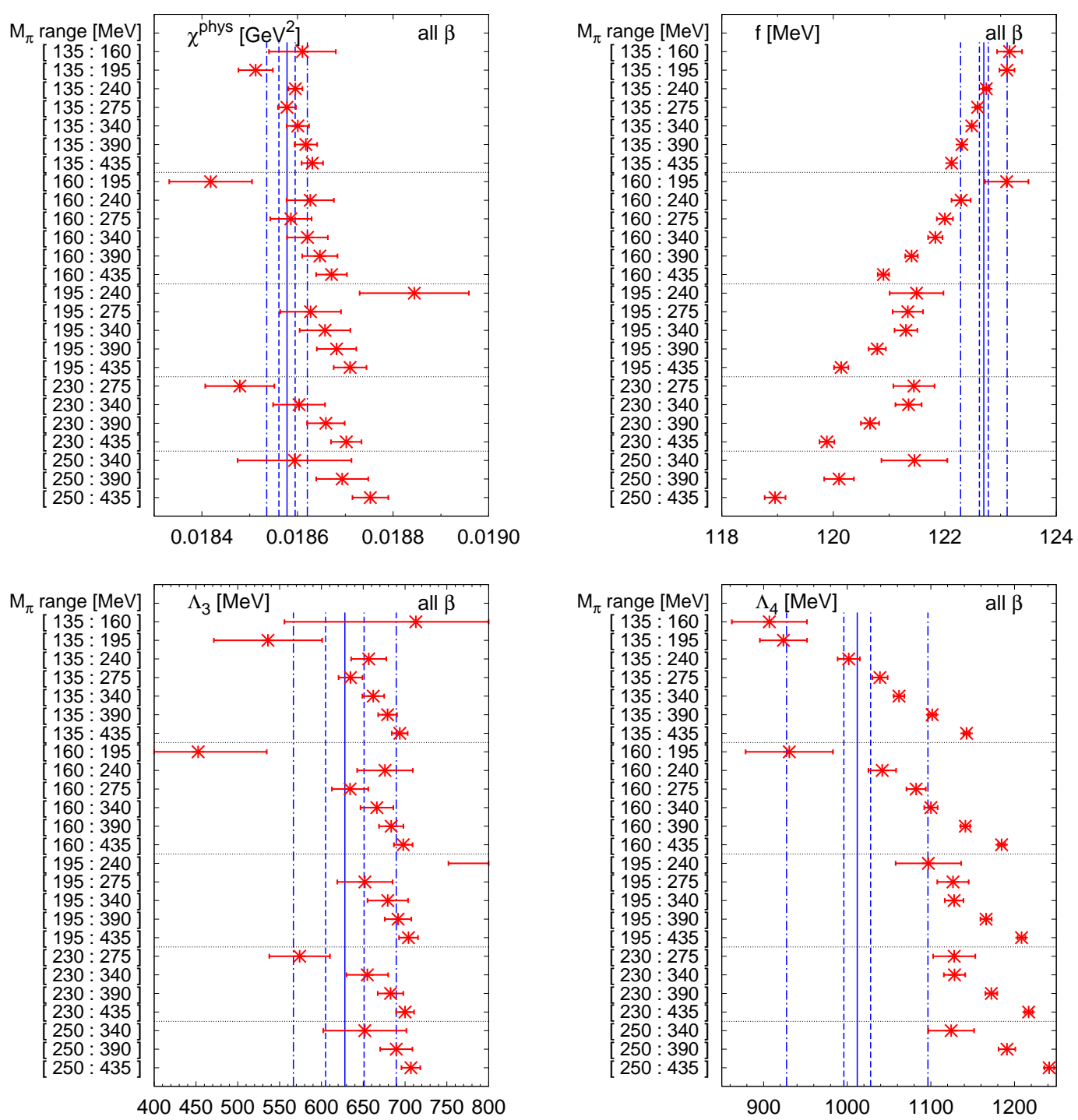

FIG. 5. Results for the fitted parameters

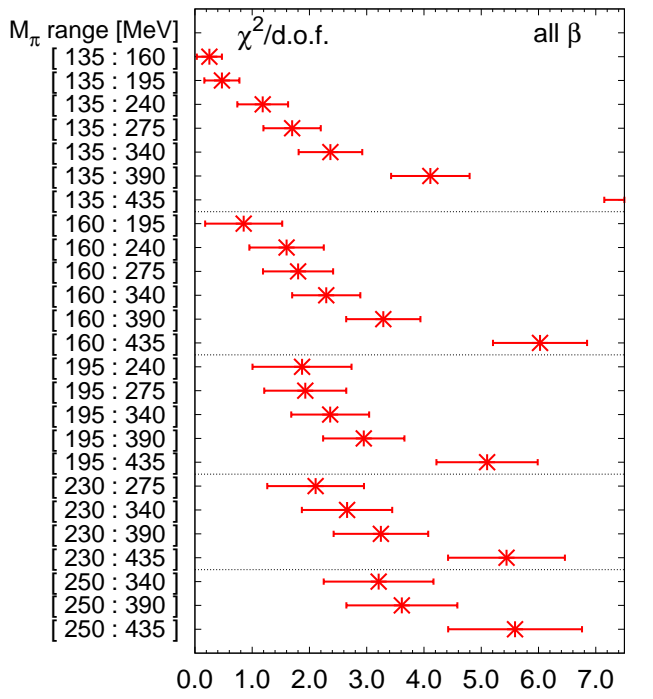

(top and middle panels) and $\chi^{2} /$ d.o.f. (bottom panel) from NLO-ChPT fits without constraints using different mass ranges but including all lattice spacings. The solid, dashed and dashed-dotted blue lines for the fit parameters denote the central value, statistical and total (statistical plus systematic) error bands, respectively, from our preferred unconstrained fit (cf. left column of Table III). 

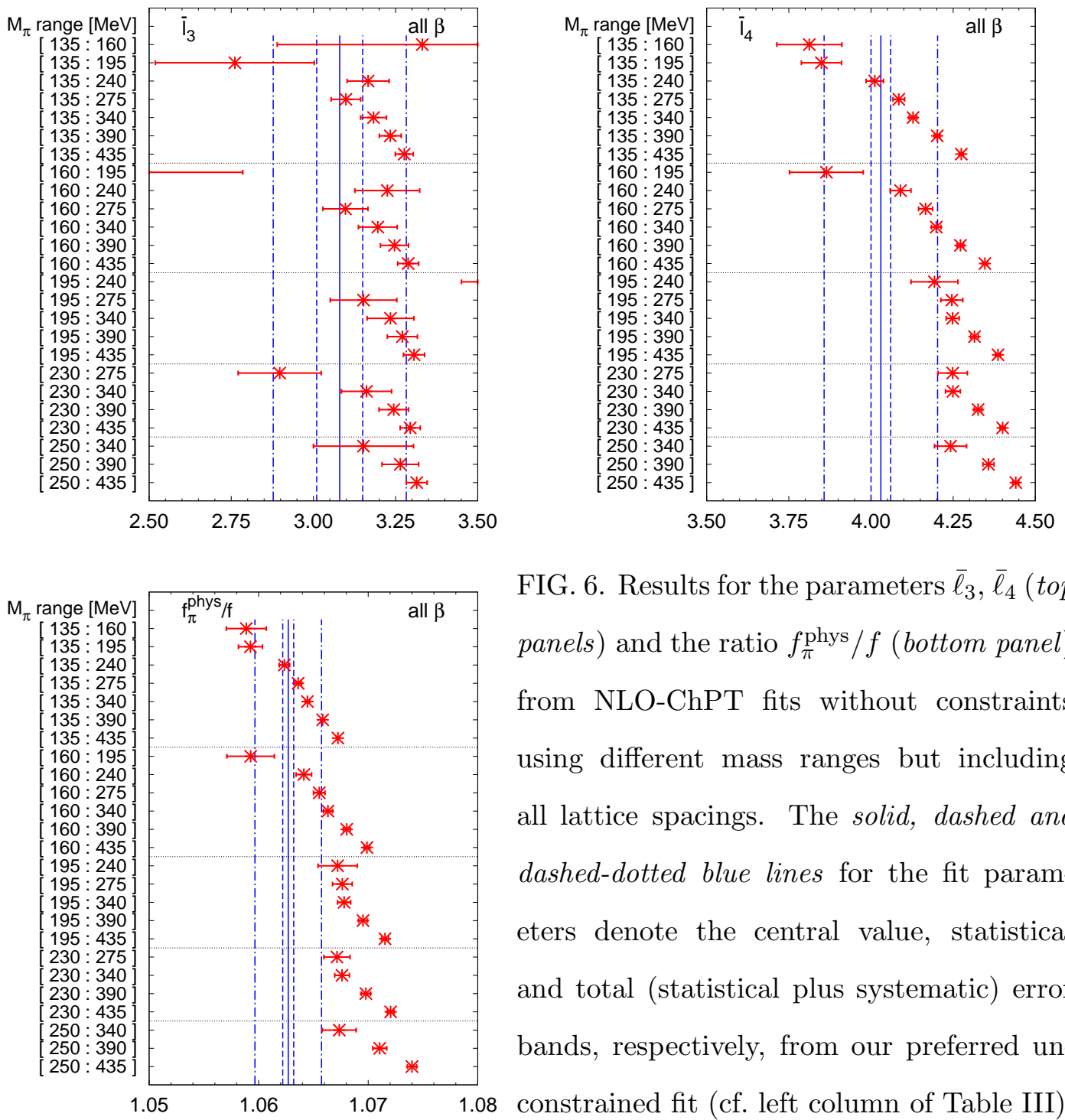

FIG. 6. Results for the parameters $\bar{\ell}_{3}, \bar{\ell}_{4}(t o p$ panels) and the ratio $f_{\pi}^{\text {phys }} / f$ (bottom panel) from NLO-ChPT fits without constraints using different mass ranges but including all lattice spacings. The solid, dashed and dashed-dotted blue lines for the fit parameters denote the central value, statistical and total (statistical plus systematic) error bands, respectively, from our preferred unconstrained fit (cf. left column of Table III).

be included. Figures 8 and 9 show the dependence of the fitted parameters and derived quantities on the range of meson masses included in the fit range. These figures should be compared with Figs. 5 and 6. Again, we observe the same pattern of reaching plateaus when excluding more and more heavier meson masses. Eventually, we decided to take the mass range $135 \mathrm{MeV} \leq M_{\pi} \leq 240 \mathrm{MeV}$ as our preferred fit, from which we will quote the central values and statistical errors. This choice is also indicated in the landscape plot, Fig. 3, by the full blue lines. The combined global fit has an acceptable $\chi^{2} /$ d.o.f. $=1.4(0.6)$ (with \#d.o.f. $=24-4=20$ ) and is shown in Fig.10, In the top panels only the data points included in the fit are plotted, while the bottom panels show the excluded data points as well. To estimate the systematic error on a fitted parameter, we take the variance 

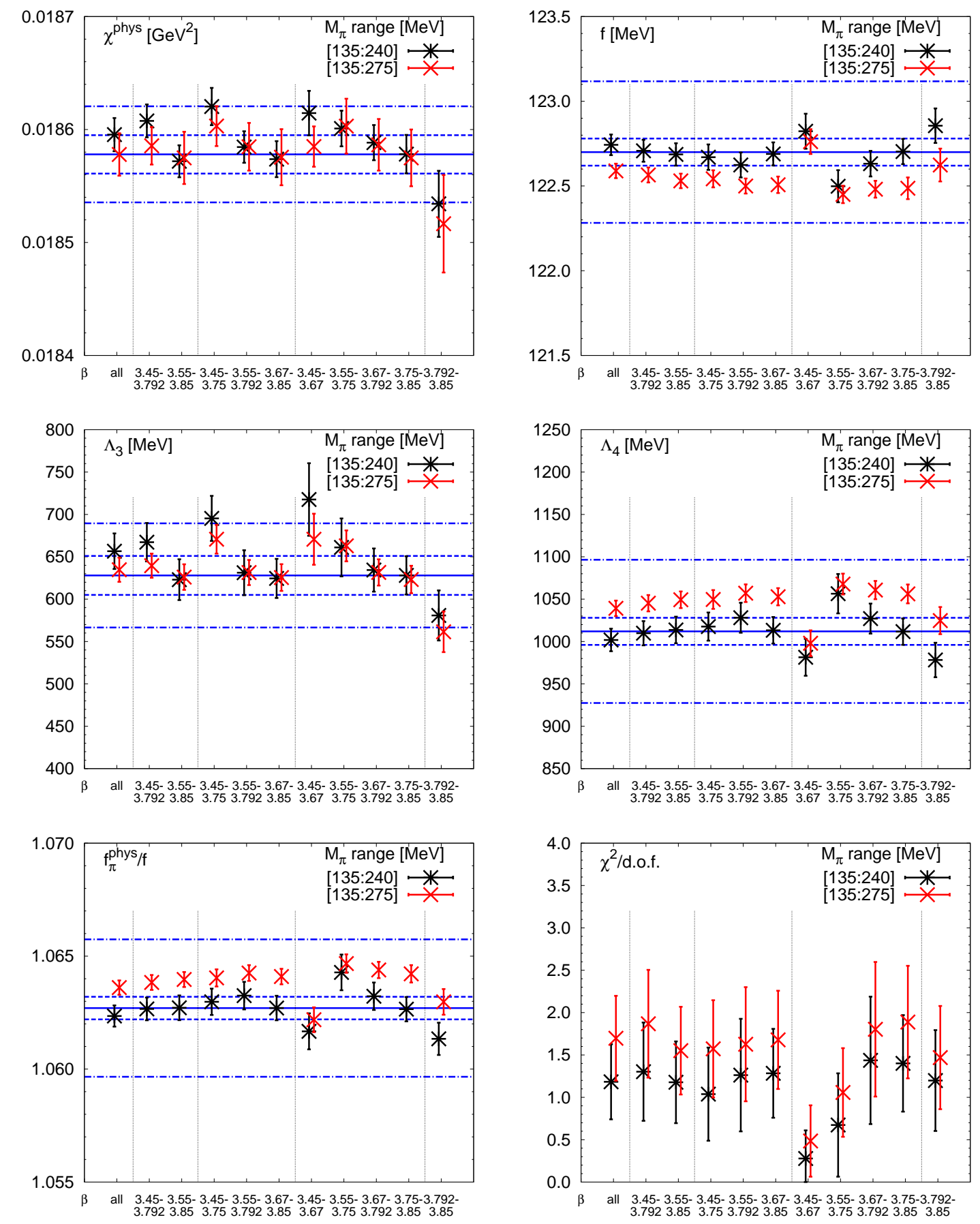

FIG. 7. Fit parameters and $\chi^{2} /$ d.o.f. from NLO-ChPT fits without constraints to two different mass ranges, where several lattice spacings have been excluded. Vertical dashed lines group points where no, one, two, three or four (from left to right) lattice spacings have been excluded. The solid, dashed and dashed-dotted horizontal blue lines denote the central value, statistical and total (statistical plus systematic) error bands, respectively, from our preferred unconstrained fit (cf. left column of Table @II). 


\begin{tabular}{lcc}
\hline \hline & unconstrained & parameter-reduced \\
\hline$\chi^{\text {phys }} /\left(10^{-2} \mathrm{GeV}^{2}\right)$ & $1.8578(17)_{\text {stat }}(39)_{\text {syst }}$ & $1.8639(18)_{\text {stat }}(44)_{\text {syst }}$ \\
$f / \mathrm{MeV}$ & $122.70(08)_{\text {stat }}(41)_{\text {syst }}$ & $122.73(06)_{\text {stat }}(28)_{\text {syst }}$ \\
$\Lambda_{3} / \mathrm{MeV}$ & $628(23)_{\text {stat }}(57)_{\text {syst }}$ & $678(40)_{\text {stat }}(119)_{\text {syst }}$ \\
$\Lambda_{4} / \mathrm{MeV}$ & $1,012(16)_{\text {stat }}(83)_{\text {syst }}$ & $1,006(15)_{\text {stat }}(71)_{\text {syst }}$ \\
$\bar{\ell}_{3}$ & $3.08(07)_{\text {stat }}(19)_{\text {syst }}$ & $3.23(12)_{\text {stat }}(30)_{\text {syst }}$ \\
$\bar{\ell}_{4}$ & $4.03(03)_{\text {stat }}(17)_{\text {syst }}$ & $4.02(03)_{\text {stat }}(14)_{\text {syst }}$ \\
$f_{\pi}^{\text {phys }} / f$ & $1.0627(05)_{\text {stat }}(30)_{\text {syst }}$ & $1.0626(06)_{\text {stat }}(24)_{\text {syst }}$ \\
\hline \hline
\end{tabular}

TABLE III. Results for LECs from unconstrained (left column, see Sec. IVB 1 for details) and parameter-reduced fits (right column, see Sec. IV B 2 for details). In the case of the unconstrained fits, the first four entries $\left(\chi^{\text {phys }}, f, \Lambda_{3}, \Lambda_{4}\right)$ are free fit parameters while the remaining entries are derived from these. For the parameter-reduced fit, only the first two entries $\left(\chi^{\text {phys }}, f\right)$ are free fit parameters. These two sets are used to calculate the final values as quoted in Eqs. (16)-(22).

of this parameter with respect to the fits using different mass ranges, which also include the near physical points. These are the topmost points above the first horizontal dashed line in each panel of Figs. 8 and 9 (as indicated by the gray shaded areas). This procedure results in the set of LECs given in the left column of Table III. Note, that only the first four parameters are fit parameters, while the remaining ones are subsequently derived from this set of parameters. The central values and error bands (statistical and combined statistical and systematic) have always been shown in the various compilations of fit results (Figs. 5 91). It is reassuring that basically all relevant results are compatible with these error bands, which a posteriori justifies our procedure of estimating the systematic error.

In the remainder of this section, we briefly discuss the influence of the near physical points on the fits and the fitted quantities (the effect of such low-end mass cuts on other observables, e.g., $m_{u d}$ or $m_{s} / m_{u d}$, have been discussed in Ref. [27]). As an example, we provide in Fig.11 the result of a combined global fit in the mass range $195 \mathrm{MeV} \leq M_{\pi} \leq 275 \mathrm{MeV}$, using ensembles with $1 / a>1.6 \mathrm{GeV}$. As can be seen from the insert magnifying the region around the physical point, for the pion decay constant the fit misses the points simulated in that region by several standard deviations. This results in an extrapolated $f_{\pi}^{\text {phys }}=$ 

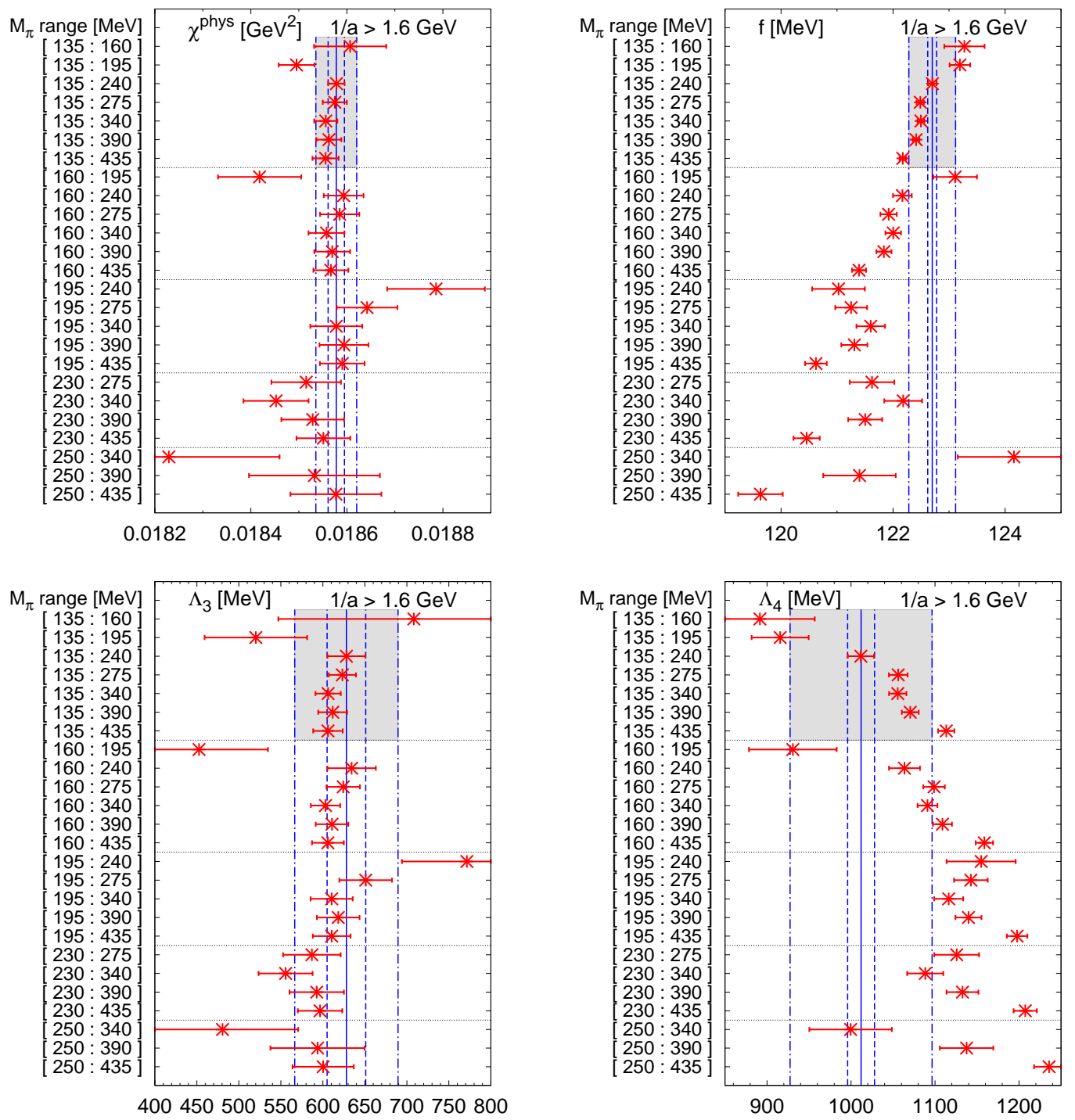

FIG. 8. Results for the fitted parameters (top and middle panels) and $\chi^{2} /$ d.o.f.

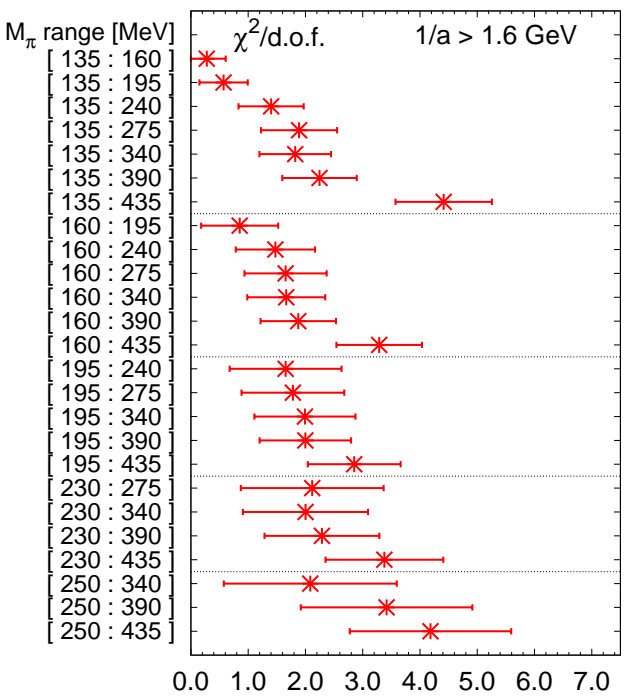
(bottom panel) from NLO-ChPT fits without constraints, using different mass ranges and including only lattice spacings $1 / a>$ 1.6 GeV. The solid, dashed and dasheddotted blue lines for the fit parameters denote the central value, statistical and total (statistical plus systematic) error bands, respectively, from our preferred unconstrained fit (cf. left column of Table ЏiI). 

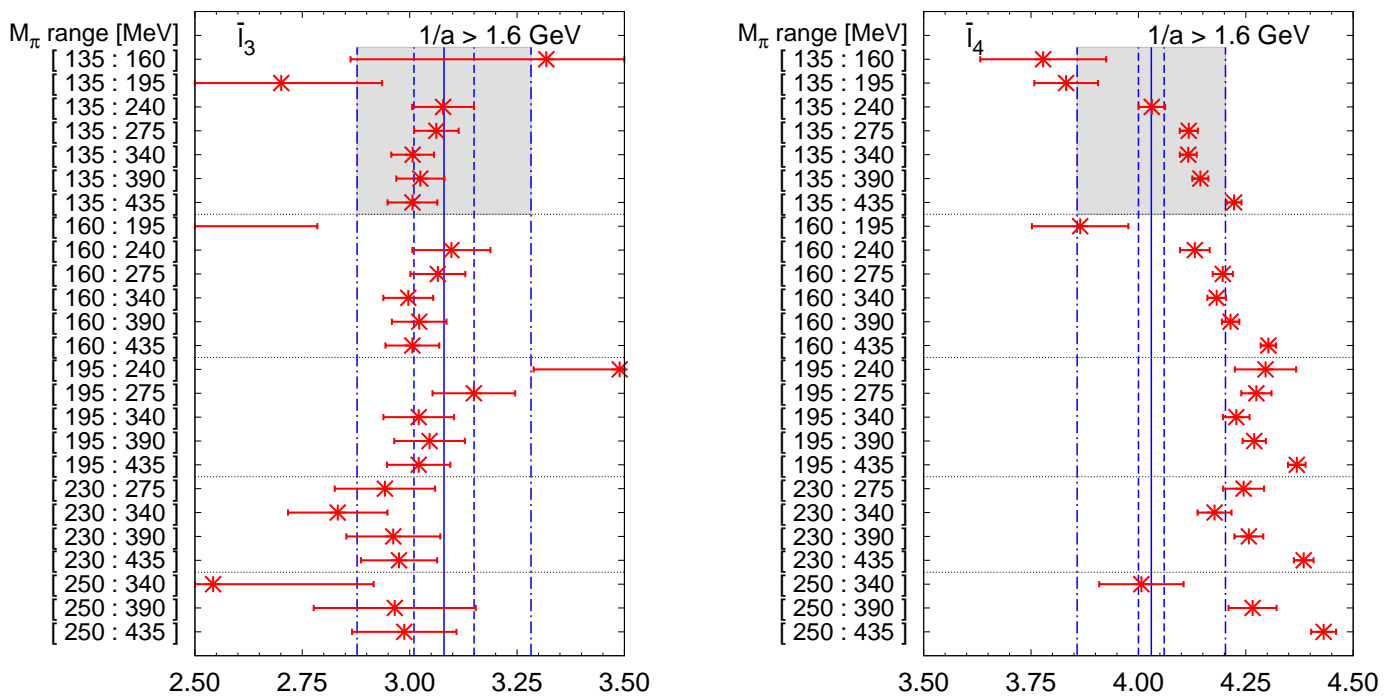

FIG. 9. Results for the parameters $\bar{\ell}_{3}, \bar{\ell}_{4}(t o p$

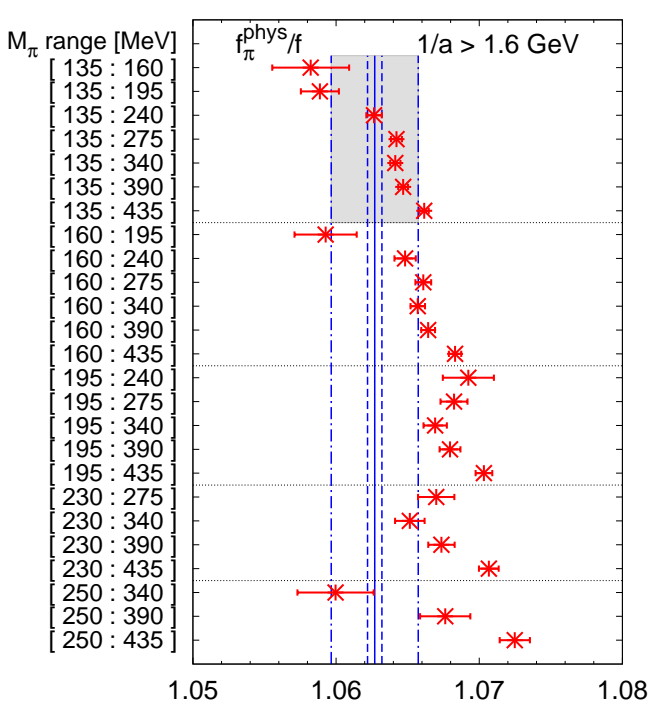
panels) and the ratio $f_{\pi}^{\text {phys }} / f$ (bottom panel) from NLO-ChPT fits without constraints, using different mass ranges and including only lattice spacings $1 / a>1.6 \mathrm{GeV}$. The solid, dashed and dashed-dotted blue lines for the fit parameters denote the central value, statistical and total (statistical plus systematic) error bands, respectively, from our preferred unconstrained fit (cf. left column of Table (II).

129.5(0.2) MeV (statistical error only), to be compared to the value of $130.41(0.03)(0.20)$ quoted by the PDG [19]. For the extrapolated pion mass the situation looks somewhat better. In Fig. 12 we provide the values for $f_{\pi}^{\text {phys }}$ and $\left(M_{\pi}^{\text {phys }}\right)^{2}$ extrapolated to the physical point by the NLO-ChPT fits with various mass ranges using ensembles with $1 / a>1.6 \mathrm{GeV}$ only. In these plots, the solid and dashed blue lines represent the central value and (total) uncertainty of these quantities as quoted by Refs. [18, 19]. Evidently, while the extrapolated pion mass is within errors compatible with the experimental result, the extrapolated pion decay constant starts to shift toward lower values once the nearly physical points are excluded from the fit range. This shift increases with both an increasing lower and higher bound on 

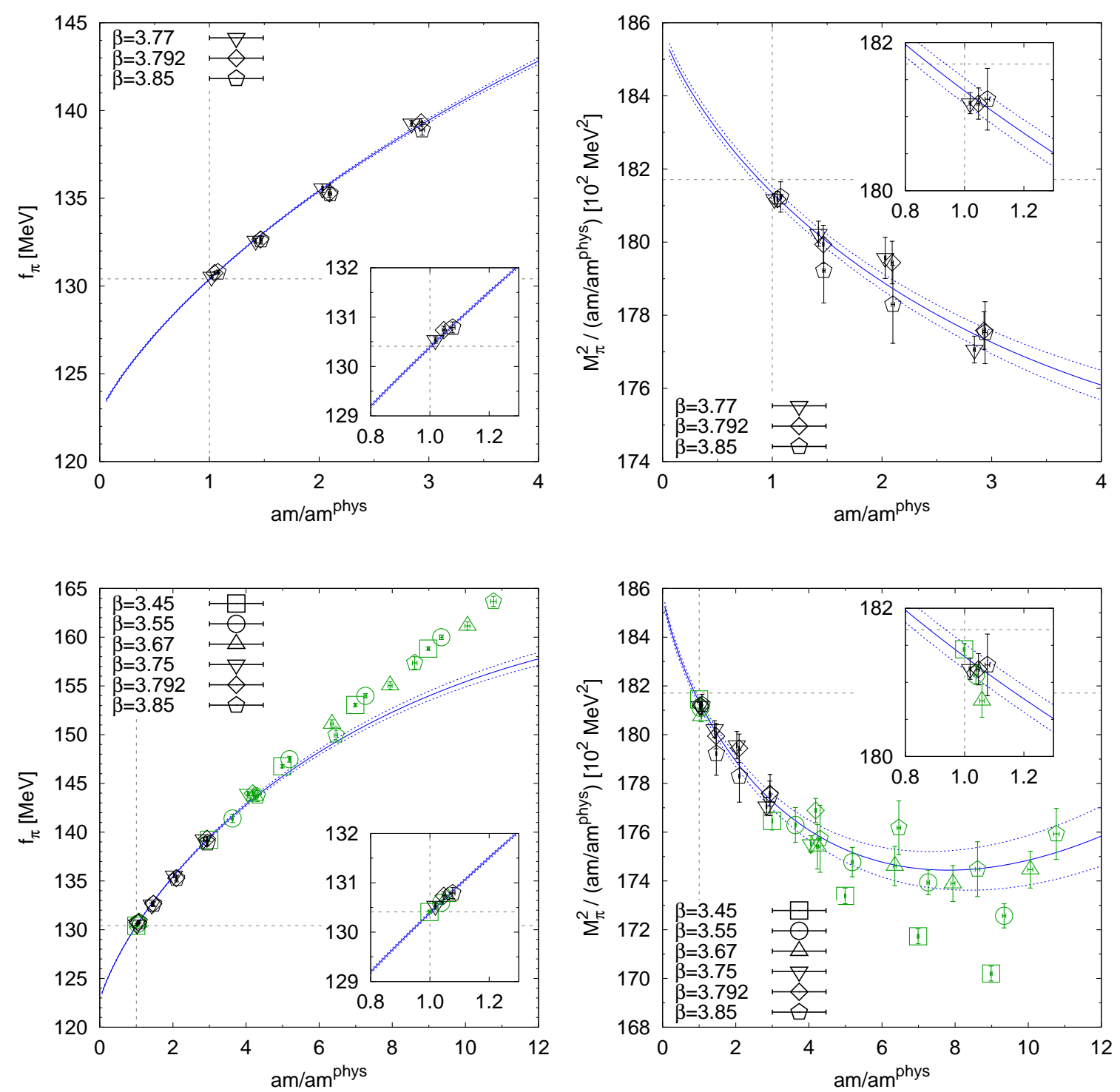

FIG. 10. Combined fit for lattice scales $1 / a>1.6 \mathrm{GeV}$ and meson masses $135 \mathrm{MeV} \leq M_{\pi} \leq$ $240 \mathrm{MeV}$. Left panels: meson decay constant, right panels: squared meson mass divided by the quark-mass ratio. The top panels show only the points, which have been included in the fit, while the bottom panels show all points. There, points marked by black symbols are included in the fit, those marked by green symbols are excluded. The physical values are indicated by dashed gray lines. 
the fit range. This behavior is also in accordance with what could be observed in the mass range plots for the fitted parameters (Figs. 5 and 8) and derived phenomenological quantities (Figs.6 and 9) shown before. These observations may be summarized as follows. For quantities like $\chi^{\text {phys }}$ and $\Lambda_{3}$ (or $\bar{\ell}_{3}$ ), which predominantly influence the quark-mass dependence of $M_{\pi}^{2}$, the values from fits excluding ensembles with low meson masses are still in agreement with our estimate. This is no longer the case for the quantities $f$ and $\Lambda_{4}$ (or $\bar{\ell}_{4}$ ) which predominantly affect the quark-mass dependence of $f_{\pi}$. It is noteworthy that the ratio $f_{\pi}^{\text {phys }} / f$ (where $f_{\pi}^{\text {phys }}$ is the value extrapolated from the fit) tends to shift toward higher values once more and more lighter masses are excluded (see, e.g., the bottom panel of Fig.9), although both $f$ and $f_{\pi}^{\text {phys }}$ are shifting toward lower values (see Figs. 8 and [12, respectively). In our opinion, these observations illustrate the danger inherent in applying NLO-ChPT formulas to lattice data with $M_{\pi}^{\text {min }}$ too large (and our data suggest that, at least for some channels, this might be the case with $M_{\pi}^{\min } \sim 200 \mathrm{MeV}$ already).

\section{Parameter-reduced NLO ChPT}

As mentioned in the beginning of this section, we also considered parameter-reduced NLO-ChPT fits which are constrained to reproduce the physical values of the pion mass and decay constant at the physical quark mass, see Eqs. (14) and (15). This will reduce the number of fit parameters in our combined global NLO fits from four to two, leaving us with $\chi^{\text {phys }}=2 B m^{\text {phys }}$ and $f$ as fit parameters in the constrained fits. Note that in this approach the low-energy constants $\bar{\ell}_{3}$ and $\bar{\ell}_{4}$ can still be determined, but they are no longer independent, since they are linked to the leading-order constants as stated in Eqs. (12) and (13). The main purpose of this exercise is to show that our unconstrained fits did not need the additional degrees of freedom to work, given that we used the pion mass and decay constants to set the scales for our lattice data. We performed fits with different mass ranges and by limiting the lattice spacings included in our fits as before. In Fig. 13 we show the results for the fitted parameters and derived quantities as well as the $\chi^{2} /$ d.o.f. for different mass ranges including only ensembles with $1 / a>1.6 \mathrm{GeV}$. The central values and error bands shown in these plots have been obtained as before: the central value and statistical error is the one from the fit with the mass range $135 \mathrm{MeV} \leq M_{\pi} \leq 240 \mathrm{MeV}$, while the systematic error has been obtained from the variation with respect to the results from 

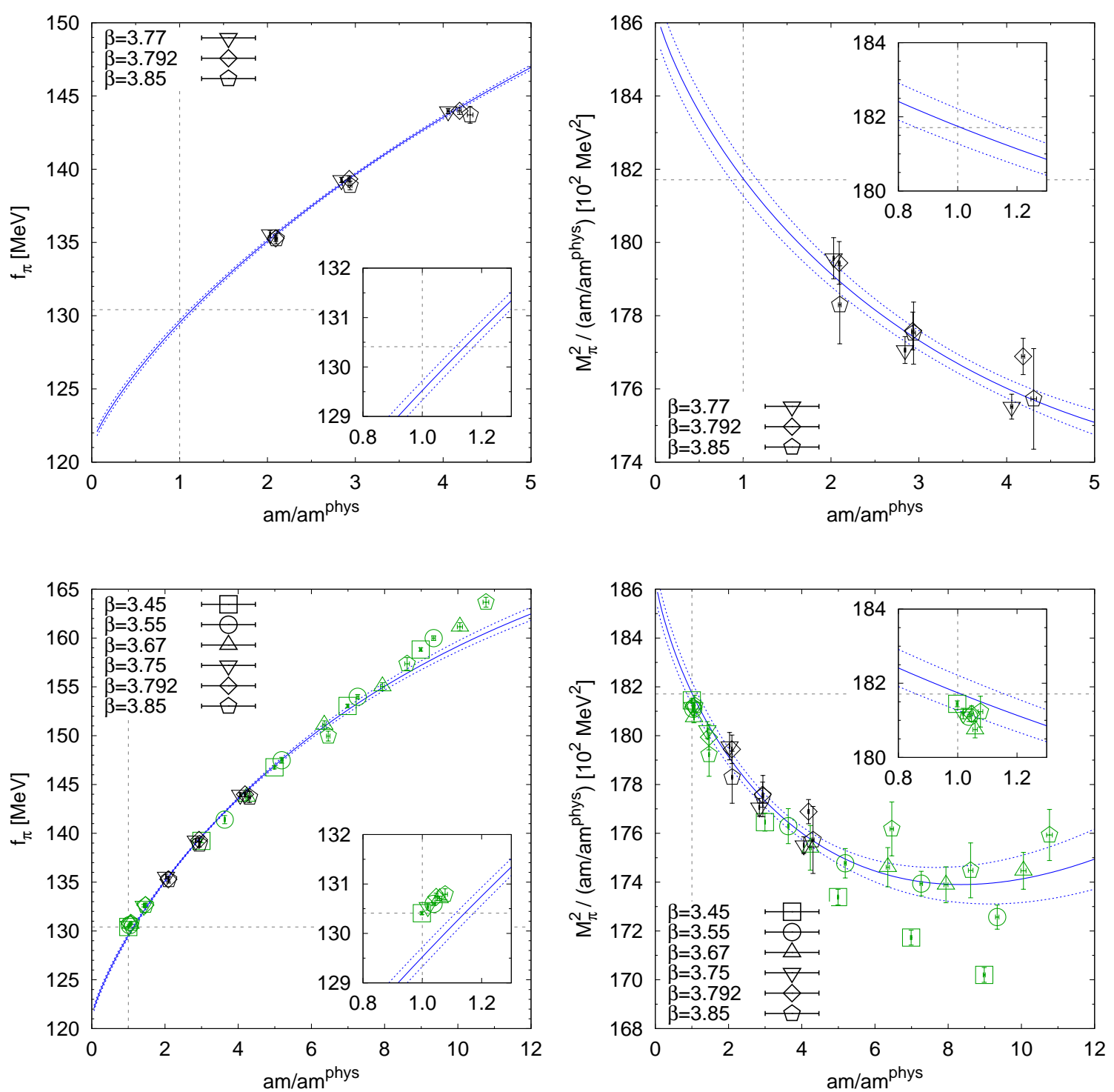

FIG. 11. Combined fit for lattice scales $1 / a>1.6 \mathrm{GeV}$ and meson masses $195 \mathrm{MeV} \leq M_{\pi} \leq$ $275 \mathrm{MeV}$ excluding the nearly physical points. Left panels: meson decay constant, right panels: squared meson mass divided by the quark-mass ratio. The top panels show only the points, which have been included in the fit, while the bottom panels show all points. There, points marked by black symbols are included in the fit, those marked by green symbols are excluded. The physical values are indicated by dashed gray lines. 

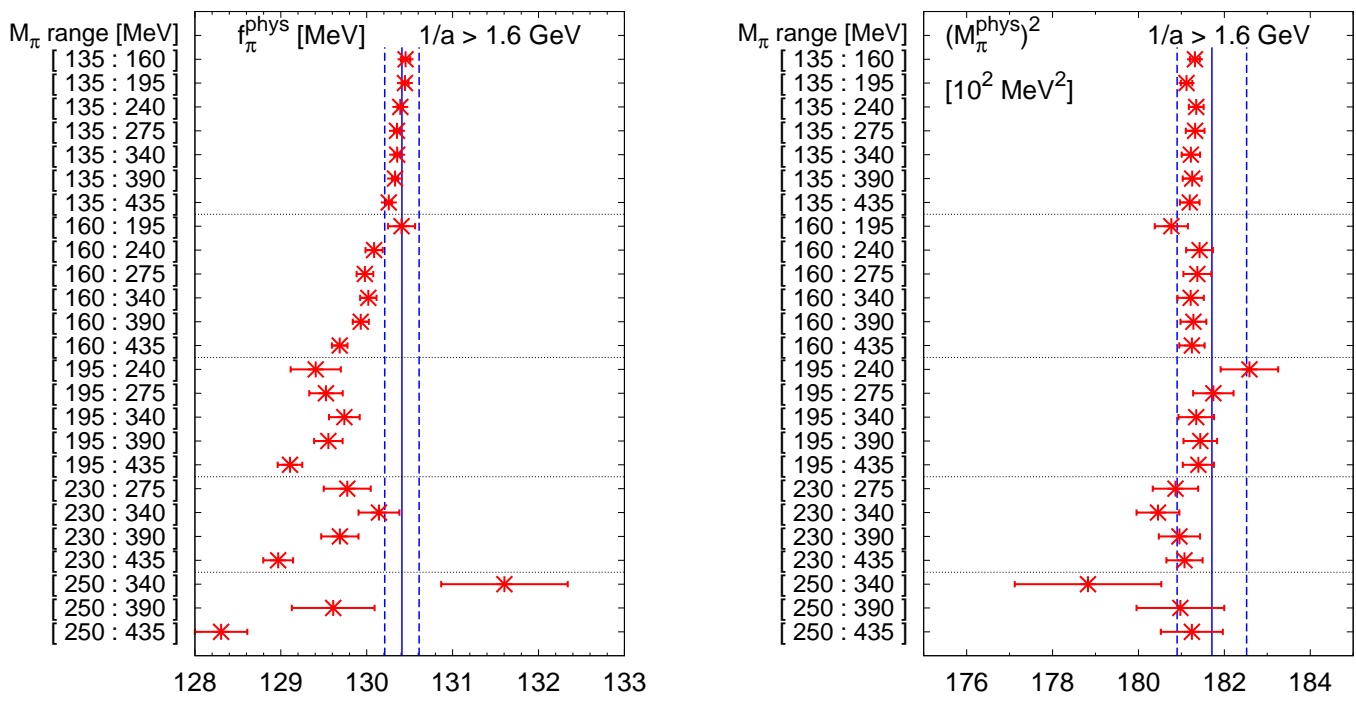

FIG. 12. Extrapolated $f_{\pi}^{\text {phys }}$ (left panel) and $\left(M_{\pi}^{\text {phys }}\right)^{2}$ (right panel) from NLO-ChPT fits without constraints, using different mass ranges and including only lattice spacings $1 / a>1.6 \mathrm{GeV}$. The solid and dashed blue lines denote the central value and total error bands, respectively, as quoted by Refs. [18, 19].

other fitting ranges in the meson mass (as indicated by the gray shaded areas). (We do not show the results from fits excluding the nearly physical points in this case, since due to the constraints these results now more or less agree with the results shown.) Figure 14 contains the plot showing the influence of the included lattice spacings on the fit results. We would like to state, that these parameter-reduced fits work equally well as the unconstrained ones, as measured by the resulting values for $\chi^{2} /$ d.o.f.. Our estimates for the LECs and derived quantities from this fitting procedure are given in the right column of Table III (the first two parameters were fitted, the remaining ones were derived from these two). These values are in good agreement with the results from the unconstrained NLO fits, given in the left column of that same table, but show a twice as large statistical and systematic error for $\Lambda_{3}$ or equivalently $\bar{\ell}_{3}$, whereas the remaining uncertainties are roughly the same or slightly reduced. 

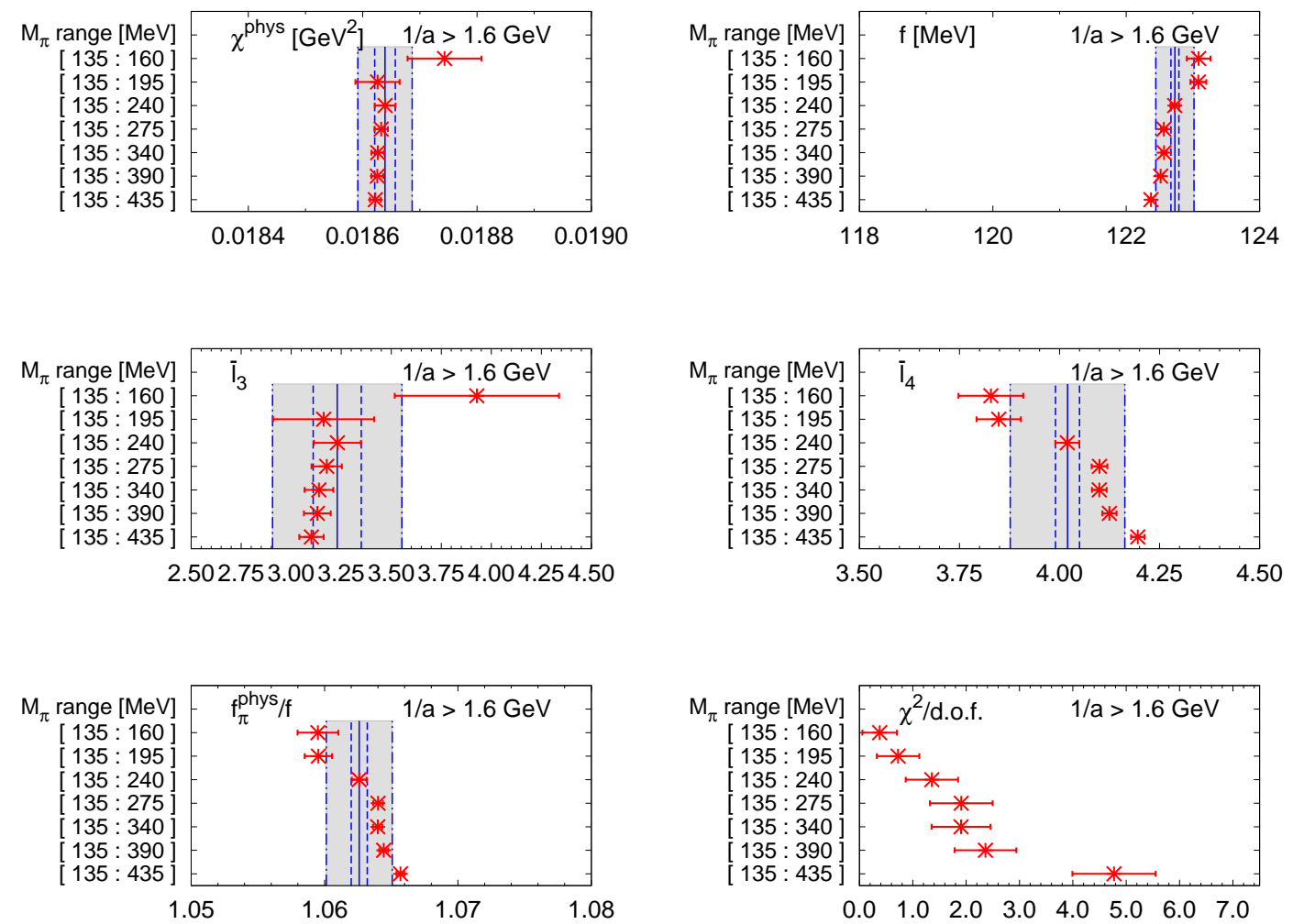

FIG. 13. Results for the fitted parameters (top panels) and $\chi^{2} /$ d.o.f. (bottom right panel) from parameter-reduced NLO-ChPT fits, using different mass ranges and including only lattice spacings $1 / a>1.6 \mathrm{GeV}$. In this setup $\bar{\ell}_{3}, \bar{\ell}_{4}$ and $f_{\pi}^{\text {phys }} / f$ are derived quantities (see text). The solid, dashed and dashed-dotted blue lines for the fit parameters denote the central value, statistical and total (statistical plus systematic) error bands, respectively, from our preferred parameter-reduced fit, cf. right column of Table III

\section{Results for LECs}

As our final set of low-energy constants determined from the NLO SU(2)-ChPT fits we quote the following values (which supersede the preliminary results in Ref. [7]).

$$
\begin{aligned}
\chi^{\text {phys }}=2 B m^{\text {phys }} & =1.8609(18)_{\text {stat }}(74)_{\text {syst }} \cdot 10^{-2} \mathrm{GeV}^{2}, \\
f & =122.72(07)_{\text {stat }}(35)_{\text {syst }} \mathrm{MeV}, \\
\Lambda_{3} & =653(32)_{\text {stat }}(101)_{\text {syst }} \mathrm{MeV}, \\
\Lambda_{4} & =1,009(16)_{\text {stat }}(77)_{\text {syst }} \mathrm{MeV},
\end{aligned}
$$



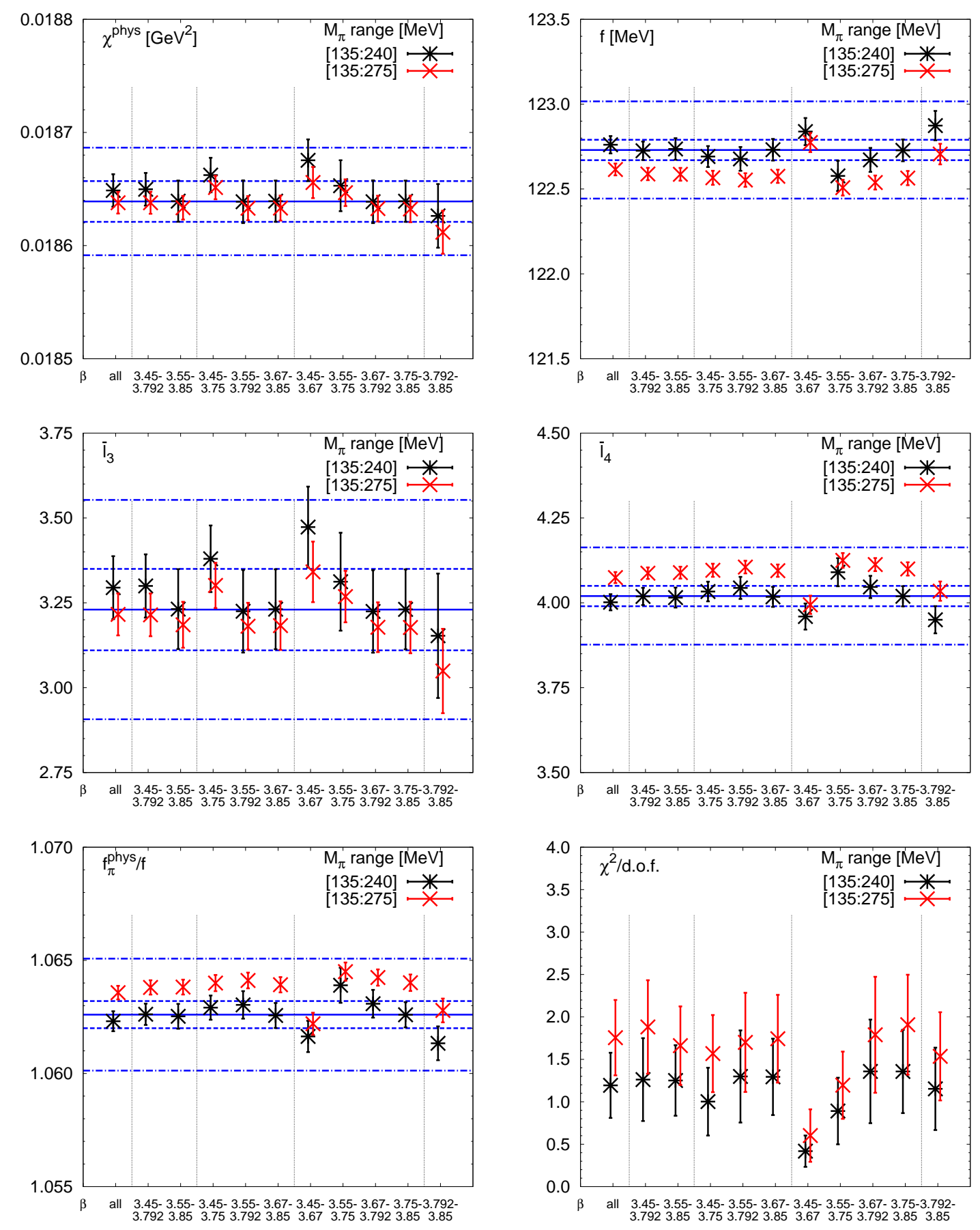

FIG. 14. Fit parameters and $\chi^{2} /$ d.o.f. from parameter-reduced NLO-ChPT fits to two different mass ranges, where several lattice spacings have been excluded. Vertical dashed lines group points where no, one, two, three or four (from left to right) lattice spacings have been excluded. The solid, dashed and dashed-dotted horizontal blue lines denote the central value, statistical and total (statistical plus systematic) error bands, respectively, from our preferred parameter-reduced fit, cf. right column of Table 【II 


$$
\begin{aligned}
\bar{\ell}_{3} & =3.16(10)_{\text {stat }}(29)_{\text {syst }}, \\
\bar{\ell}_{4} & =4.03(03)_{\text {stat }}(16)_{\text {syst }}, \\
f_{\pi}^{\text {phys }} / f & =1.0627(06)_{\text {stat }}(27)_{\text {syst }} .
\end{aligned}
$$

Here we averaged the central values and statistical uncertainties from the unconstrained (Sec. IV B 1) and parameter-reduced (Sec. IV B 2) fits (both summarized in Table III). For the square of the systematic uncertainty, we sum the squares of the average systematic uncertainty and of the spread of the central values.

\section{FITS TO NNLO-SU(2) CHPT}

As a further step, we examined the effects of fitting our data to $\mathrm{SU}(2)$ ChPT including terms up to NNLO. The motivation for this exercise is twofold. On the one hand, we would like to see whether our results obtained for the LECs by fitting to NLO ChPT are stable when considering the next-higher order in ChPT. On the other hand, there is some general interest in whether the amount of data is sufficient to reliably determine the additional fit parameters that appear at NNLO and whether NNLO ChPT is superior to NLO-ChPT in terms of describing the data (as measured, e.g., by the $\chi^{2} /$ d.o.f.).

\section{A. Fit formulas}

The formulas for the squared meson mass and the meson decay constant up to NNLO read (cf. Refs. [18, 28])

$$
\begin{aligned}
M_{\pi}^{2} & =\chi\left[1+\frac{\chi}{16 \pi^{2} f^{2}} \log \frac{\chi}{\Lambda_{3}^{2}}+\mathrm{NNLO}_{M^{2}}\right] \\
f_{\pi} & =f\left[1-\frac{\chi}{8 \pi^{2} f^{2}} \log \frac{\chi}{\Lambda_{4}^{2}}+\mathrm{NNLO}_{f}\right] \\
\mathrm{NNLO}_{M^{2}} & =\left(\frac{\chi}{16 \pi^{2} f^{2}}\right)^{2}\left[\frac{1}{306}\left(60 \log \frac{\chi}{\Lambda_{12}^{2}}-9 \log \frac{\chi}{\Lambda_{3}^{2}}-49\right)^{2}+4 k_{M^{2}}\right] \\
\mathrm{NNLO}_{f} & =\left(\frac{\chi}{16 \pi^{2} f^{2}}\right)^{2}\left[-\frac{1}{180}\left(30 \log \frac{\chi}{\Lambda_{12}^{2}}+6 \log \frac{\chi}{\Lambda_{3}^{2}}-6 \log \frac{\chi}{\Lambda_{4}^{2}}-23\right)^{2}+4 k_{f}\right]
\end{aligned}
$$

Up to this order three new fit parameters enter: one additional low-energy scale $\Lambda_{12}$ and the two NNLO LECs $k_{M^{2}}, k_{f}$. The low-energy scale $\Lambda_{12}$ is related to the low-energy scales 
usually denoted by $\Lambda_{1}$ and $\Lambda_{2}$ in the literature via

$$
\log \Lambda_{12}^{2}=\frac{7}{15} \log \Lambda_{1}^{2}+\frac{8}{15} \log \Lambda_{2}^{2}
$$

The low-energy scales $\Lambda_{1}, \Lambda_{2}$ already appear separately in the NLO formulas for other quantities (e.g., scattering lengths in $\pi \pi$ scattering [28]), but since in our case only the combination $\Lambda_{12}$ appears, we will not be able to distinguish between them. As before, the low-energy scales can also be expressed via the LECs $\bar{\ell}_{i}$ as in Eq. (10), i.e.

$$
\bar{\ell}_{i}=\log \frac{\Lambda_{i}^{2}}{\left(M_{\pi}^{\text {phys }}\right)^{2}}, \quad i=1,2,3,4,12 .
$$

\section{B. Combined global fits}

We applied the same fit strategy for the NNLO fits as for the NLO fits (see the previous section): the scale and light quark mass at the physical point as determined in Sec.III are used, and combined global fits to our data for the meson masses and decay constants are performed. First, we will discuss fits without any assumptions on the fit parameters (Sec.VB 1). Later we will also constrain the additional fit parameters entering at NNLO by phenomenologically motivated estimates (Sec.VB2).

\section{NNLO fits without priors}

We will start our discussion with fit ranges including the nearly physical points and only consider varying the upper mass limit of the fit range. Since now three more parameters have to be determined, it can be expected that we will have to include more data points, i.e., including higher meson masses, compared to the NLO case. Indeed, with too few data points either the fitter could not find a solution at all or some of the fitted parameters had big numerical uncertainties. This can be seen in the compilation in Fig.[15 of fit results using ensembles with $1 / a>1.6 \mathrm{GeV}$, e.g., for the fit ranges $135 \mathrm{MeV} \leq M_{\pi} \leq 240 \mathrm{MeV}$, $135 \mathrm{MeV} \leq M_{\pi} \leq 275 \mathrm{MeV}$ and maybe also $135 \mathrm{MeV} \leq M_{\pi} \leq 340 \mathrm{MeV}$.

The plots of a sample fit with the range $135 \mathrm{MeV} \leq M_{\pi} \leq 390 \mathrm{MeV}, 1 / a>1.6 \mathrm{GeV}$ are given in Fig.16 with a breakup into LO, NLO, and NNLO contributions (only data points included in the fit range are shown in these plots). Although technically the fit seems to work 

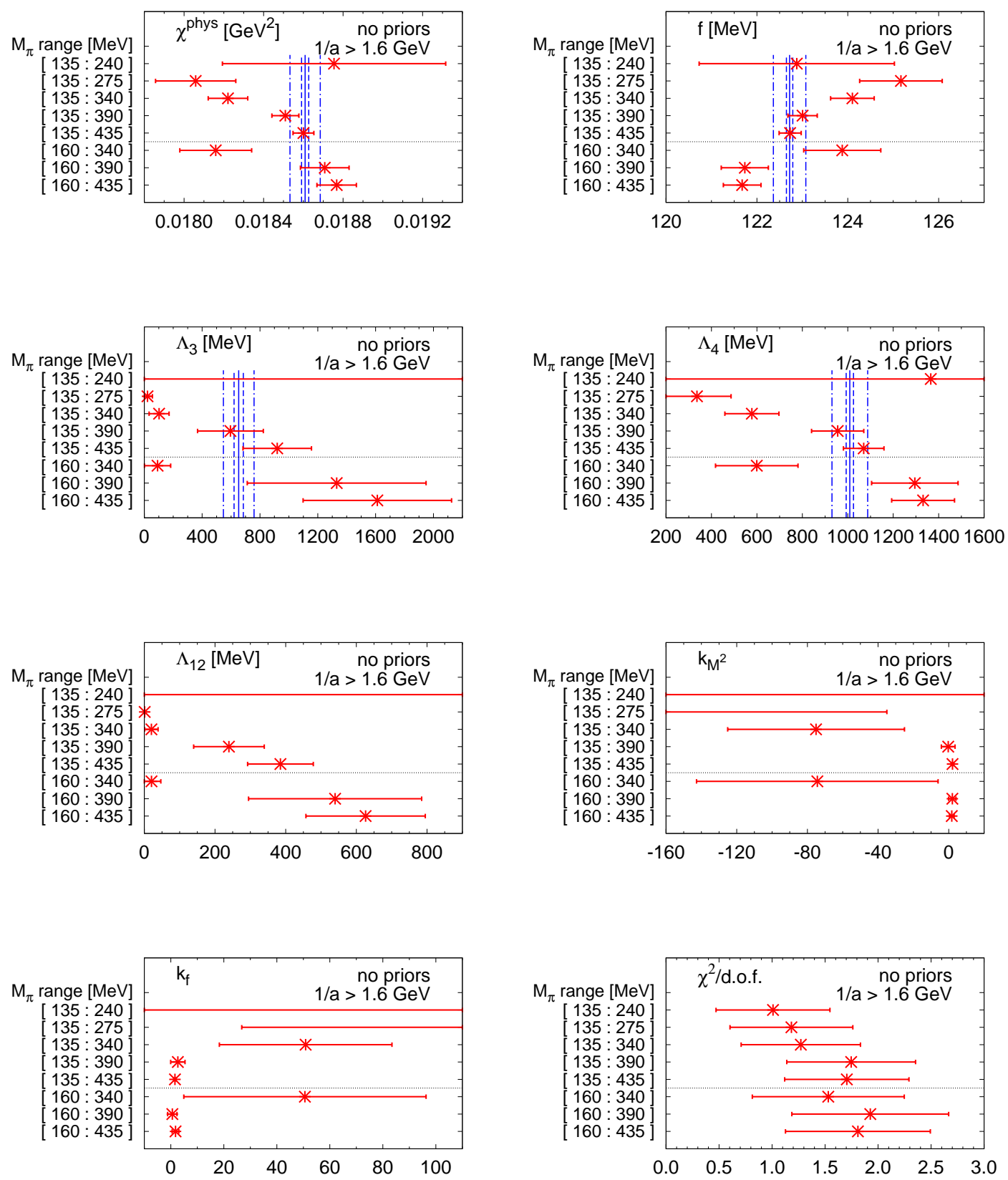

FIG. 15. Results for the fitted parameters and $\chi^{2} /$ d.o.f. from NNLO ChPT fits without constraints using different mass ranges and including lattice spacings $1 / a>1.6 \mathrm{GeV}$. The solid, dashed and dashed-dotted blue lines for the fit parameters denote the central value, statistical and total (statistical plus systematic) error bands, respectively, from our NLO fits, cf. Eqs. (16)-(22). 

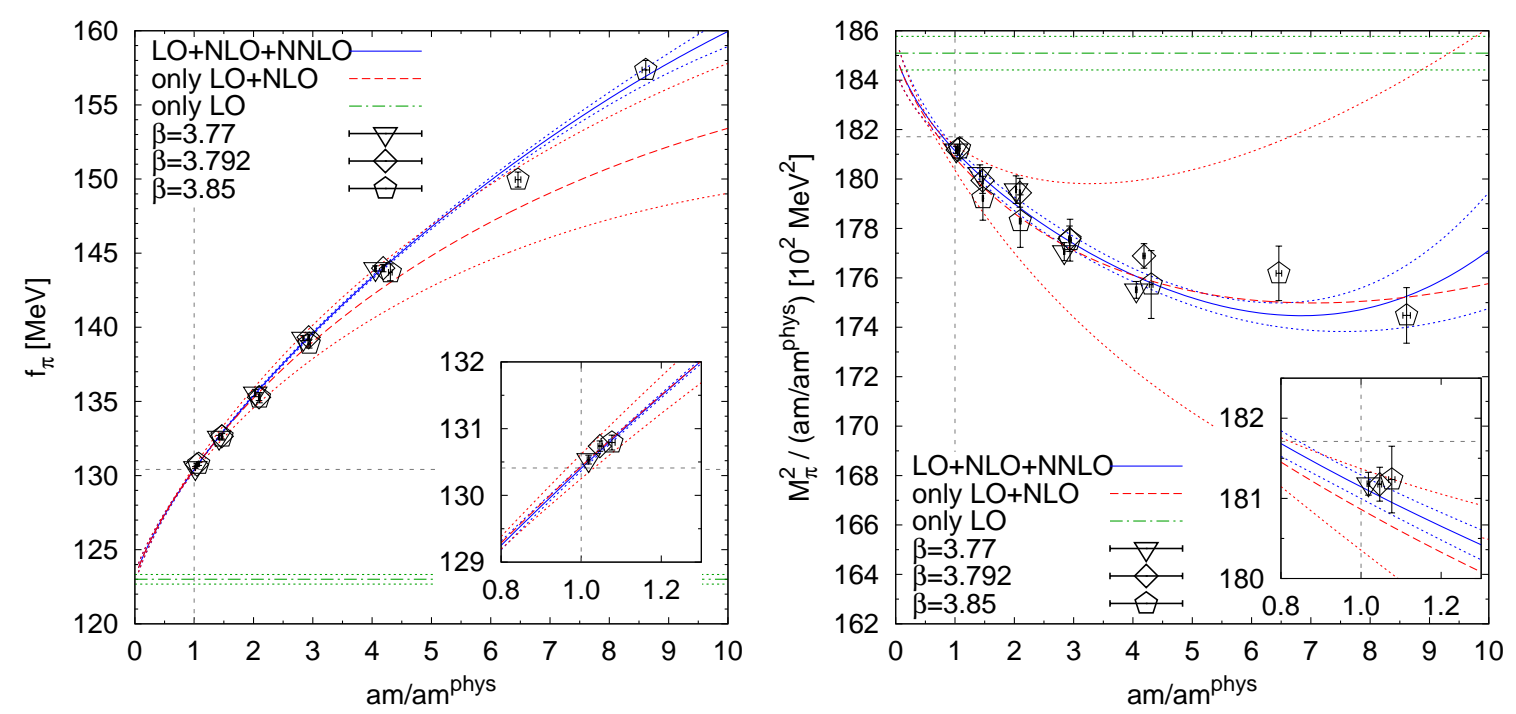

FIG. 16. Combined NNLO fit for lattice scales $1 / a>1.6 \mathrm{GeV}$ and meson masses $135 \mathrm{MeV} \leq$ $M_{\pi} \leq 390 \mathrm{MeV}$. Left panel: meson decay constant, right panel: squared meson mass divided by the quark-mass ratio. The solid blue lines show the complete (up to NNLO) fit, whereas the dashed red lines show the LO+NLO contribution of the full NNLO fit, and the dash-dotted green lines show the LO contribution. Only data points included in the fit range are depicted in the plots, the physical values are marked by dashed gray lines.

well, resulting in an acceptable $\chi^{2} /$ d.o.f. $=1.8(0.6)$ (with $\#$ d.o.f. $=34-7=27$ ) one should take the result with a grain of salt. Besides the fitted curve up to NNLO (solid blue line with error band indicated by blue dotted lines), each plot also shows the LO+NLO contribution separately (dashed red line with error band indicated by red dotted lines), i.e., the NNLO contribution being the difference between the solid blue and the dashed red line. In this case, the NLO and NNLO contributions taken separately seem to have big uncertainties. In other cases, we found the NNLO contribution to have an unnaturally large effect even at small quark masses.

At this point we have to conclude that NNLO fits to our current data are not convincing for the reasons outlined above. This situation might improve once more data points in the region between the physical point (or below) and, say, 200 or $250 \mathrm{MeV}$ will be added to the analysis, allowing for fits using a smaller mass range (like we were able to do for the NLO fits). Since the generation of such data points is not planned for the near future, we 
will in the remainder of this section examine whether constraining the additional NNLO fit parameters can serve as a remedy to this situation (and which side effects this remedy has).

\section{NNLO fits using priors}

To stabilize the NNLO fits, we examined the effect of using priors for the three additional fit parameters $\Lambda_{12}, k_{M^{2}}$, and $k_{f}$. Since we are mainly interested to learn how the parameters already appearing at NLO change, when going from NLO to NNLO fits, we did not consider adding priors for these parameters as well.

The priors were implemented in the fitting procedure via an augmented $\chi^{2}$. Instead of minimizing the usual $\chi^{2}$ (sum of deviations of the data points from the fitted function weighted by the uncertainty of that data point) the modified

$$
\chi_{\text {augmented }}^{2}=\chi^{2}+\sum_{n} \frac{\left(p_{n}-p_{n}^{\text {prior }}\right)^{2}}{\left(\sigma_{n}^{\text {prior }}\right)^{2}}
$$

is minimized by the fitting routine. Here $n$ runs over all fit parameters $p_{n}$ to which a prior $p_{n}^{\text {prior }}$ with width $\sigma_{n}^{\text {prior }}$ has been assigned. That is, the fitting routine "punishes" a parameter for leaving the prior interval $p_{n}^{\text {prior }} \pm \sigma_{n}^{\text {prior }}$.

In the following we will use either a prior for $\bar{\ell}_{12}$ alone or together with priors for $k_{M^{2}}$ and $k_{f}$. We will assign the following values for the priors to the fit parameters:

$$
\begin{aligned}
& \bar{\ell}_{12}^{\text {prior }}=2.1 \pm 0.3 \\
& k_{M^{2}}^{\text {prior }}=0 \pm 10 \\
& k_{f}^{\text {prior }}=0 \pm 10 .
\end{aligned}
$$

The prior for $\bar{\ell}_{12}$ has been obtained from the estimates for the LECs $\bar{\ell}_{1}=-0.4 \pm 0.6$ and $\bar{\ell}_{2}=4.3 \pm 0.1$ extracted from $\pi \pi$-scattering data in Ref. [28] via

$$
\bar{\ell}_{12}^{\text {prior }}=\frac{7}{15} \bar{\ell}_{1}^{\text {prior }}+\frac{8}{15} \bar{\ell}_{2}^{\text {prior }}
$$

cf. Eqs. (27) and (28). This would translate into a prior for the low-energy scale

$$
\Lambda_{12}^{\text {prior }}=385 \mathrm{MeV} \pm 58 \mathrm{MeV} .
$$

The priors for the NNLO LECs $k_{M^{2}}$ and $k_{f}$ are merely based on assuming a natural order of magnitude for these corrections. We are aware that the latter is a rather weak argument, 

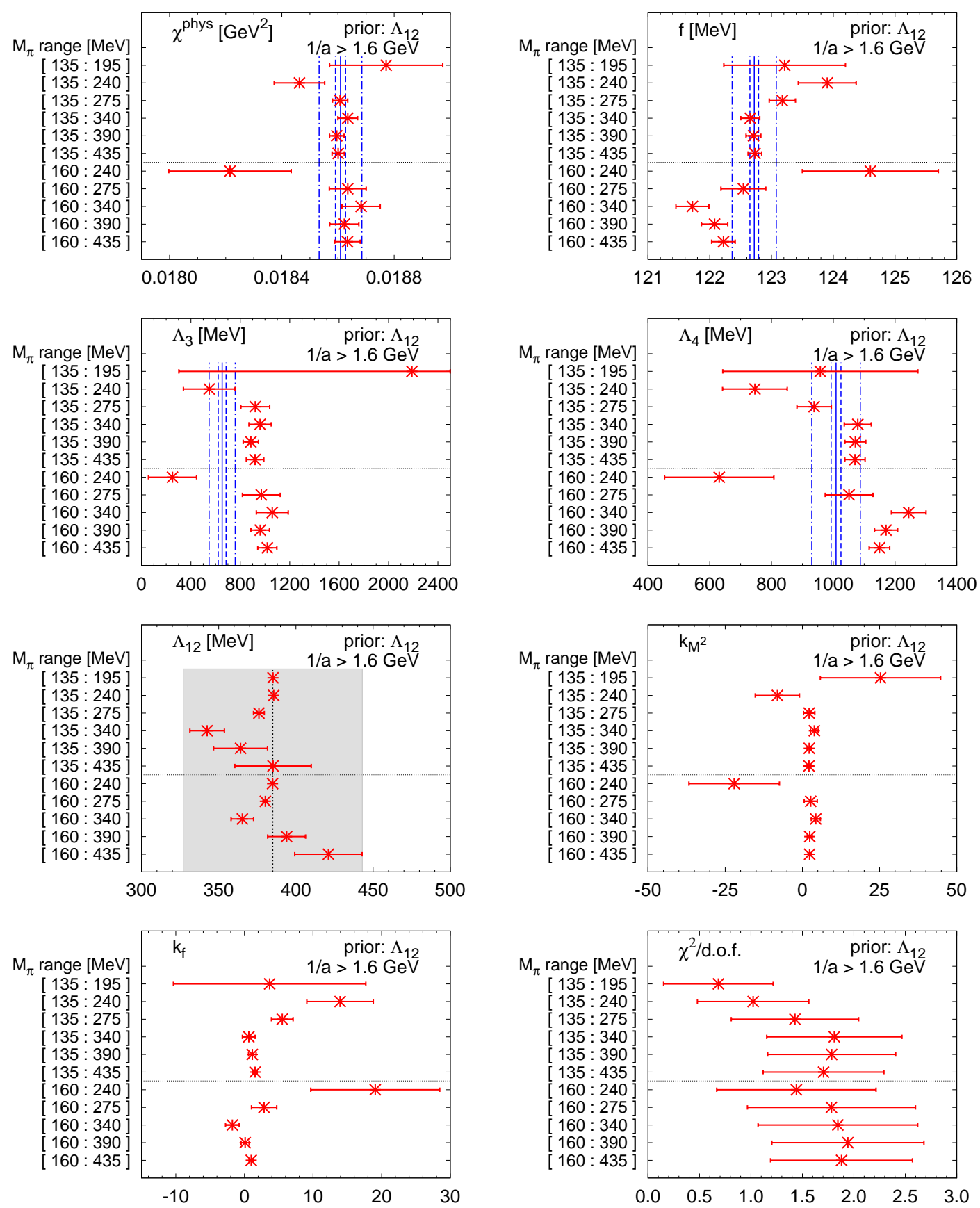

FIG. 17. Results for the fitted parameters and $\chi^{2} /$ d.o.f. from NNLO-ChPT fits with a prior for $\Lambda_{12}$ using different mass ranges and including lattice spacings $1 / a>1.6 \mathrm{GeV}$. The solid, dashed and dashed-dotted blue lines for the fit parameters denote the central value, statistical and total (statistical plus systematic) error bands, respectively, from our NLO fits, cf. Eqs. (16)-(22). The prior on $\Lambda_{12}$ and its width are indicated by the shaded gray area in the respective panel. 

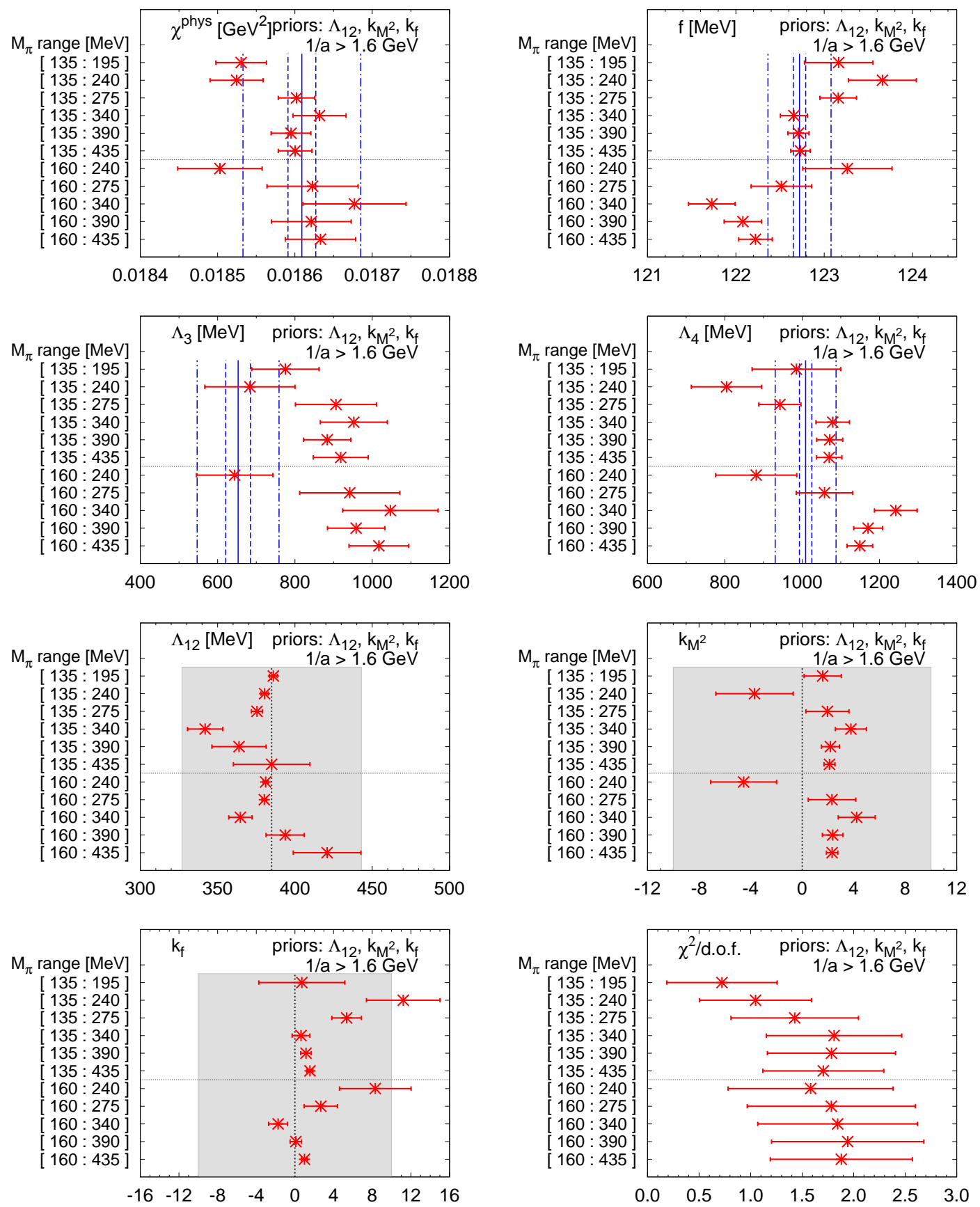

FIG. 18. Results for the fitted parameters and $\chi^{2} /$ d.o.f. from NNLO-ChPT fits with priors for $\Lambda_{12}$, $k_{M^{2}}$, and $k_{f}$ using different mass ranges and including lattice spacings $1 / a>1.6 \mathrm{GeV}$. The solid, dashed and dashed-dotted blue lines for the fit parameters denote the central value, statistical and total (statistical plus systematic) error bands, respectively, from our NLO fits, cf. Eqs. (16)-(22). The priors and their widths are indicated by the shaded gray areas in the respective panels. 

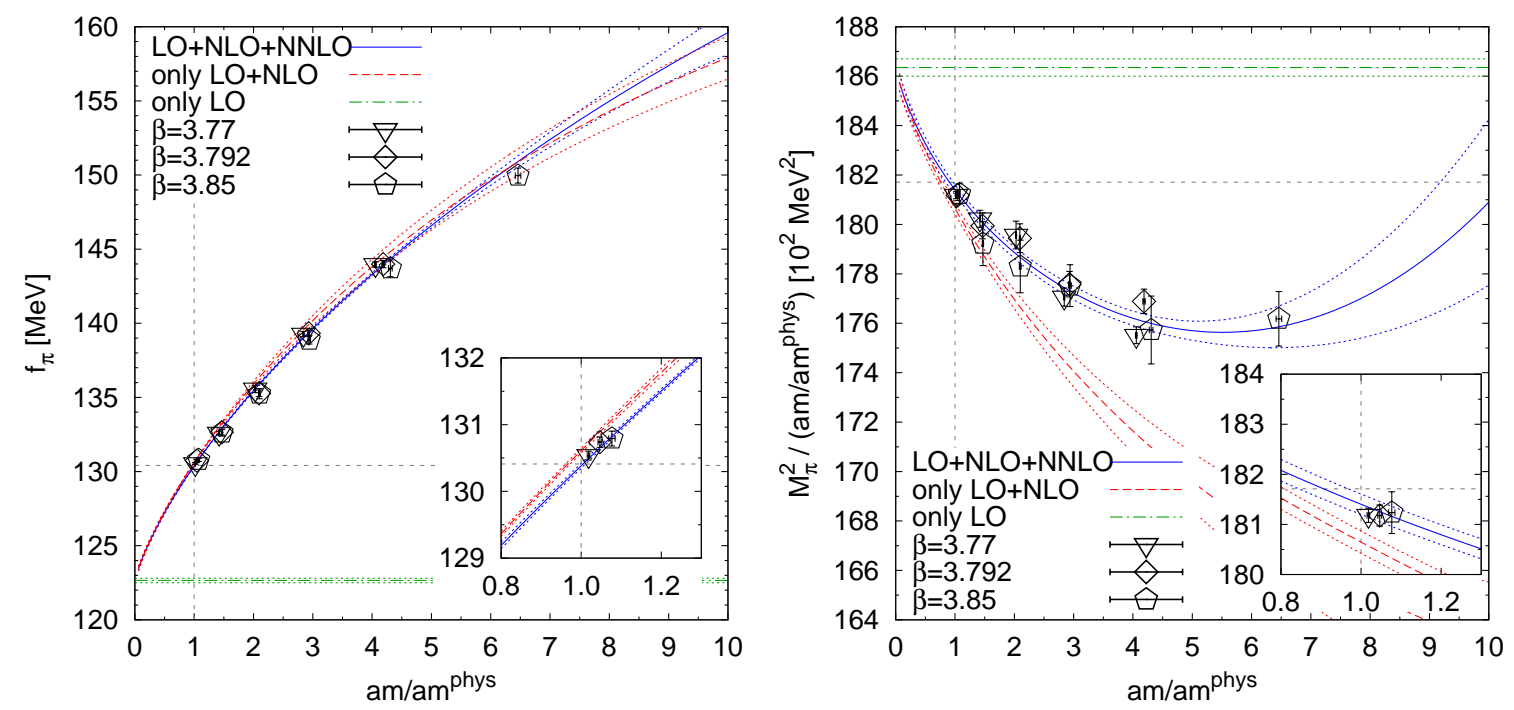

FIG. 19. Combined NNLO fit for lattice scales $1 / a>1.6 \mathrm{GeV}$ and meson masses $135 \mathrm{MeV} \leq$ $M_{\pi} \leq 340 \mathrm{MeV}$ using a prior for $\Lambda_{12}$. Left panel: meson decay constant, right panel: squared meson mass divided by the quark-mass ratio. The solid blue lines show the complete (up to NNLO) fit, whereas the dashed red lines show the LO+NLO contribution of the full NNLO fit, and the dash-dotted green lines show the LO contribution. Only data points included in the fit range are depicted in the plots; the physical values are marked by dashed gray lines.

for that reason we did not use the priors on $k_{M^{2}}$ and $k_{f}$ alone, and results from fits using these priors should be taken with some caution. However, these priors are to some extent justified by the NNLO fits where no priors have been used (see Fig.15) and also, as we will see, by fits where only the prior on $\bar{\ell}_{12}$ has been used.

The results from the fits to different mass ranges using priors on $\bar{\ell}_{12}$ only or on $k_{M^{2}}$ and $k_{f}$ as well are shown in Figs.17 and 18, respectively, where only the ensembles with $1 / a>1.6 \mathrm{GeV}$ have been used. The used priors are indicated by the gray shaded areas. As an effect, now fits to smaller mass ranges are possible and/or are more stable judging from the uncertainties of the fit parameters. In Figs.19] and 20, we show as examples the fits for the mass range $135 \mathrm{MeV} \leq M_{\pi} \leq 340 \mathrm{MeV}$, with a breakup into LO, NLO, and NNLO contributions. Whereas in both cases for the decay constant the NNLO contribution seems to be reasonably small, for the meson mass, the NNLO contribution is more substantial. Nevertheless, the error bands for the LO+NLO contribution are now reasonable as well, 

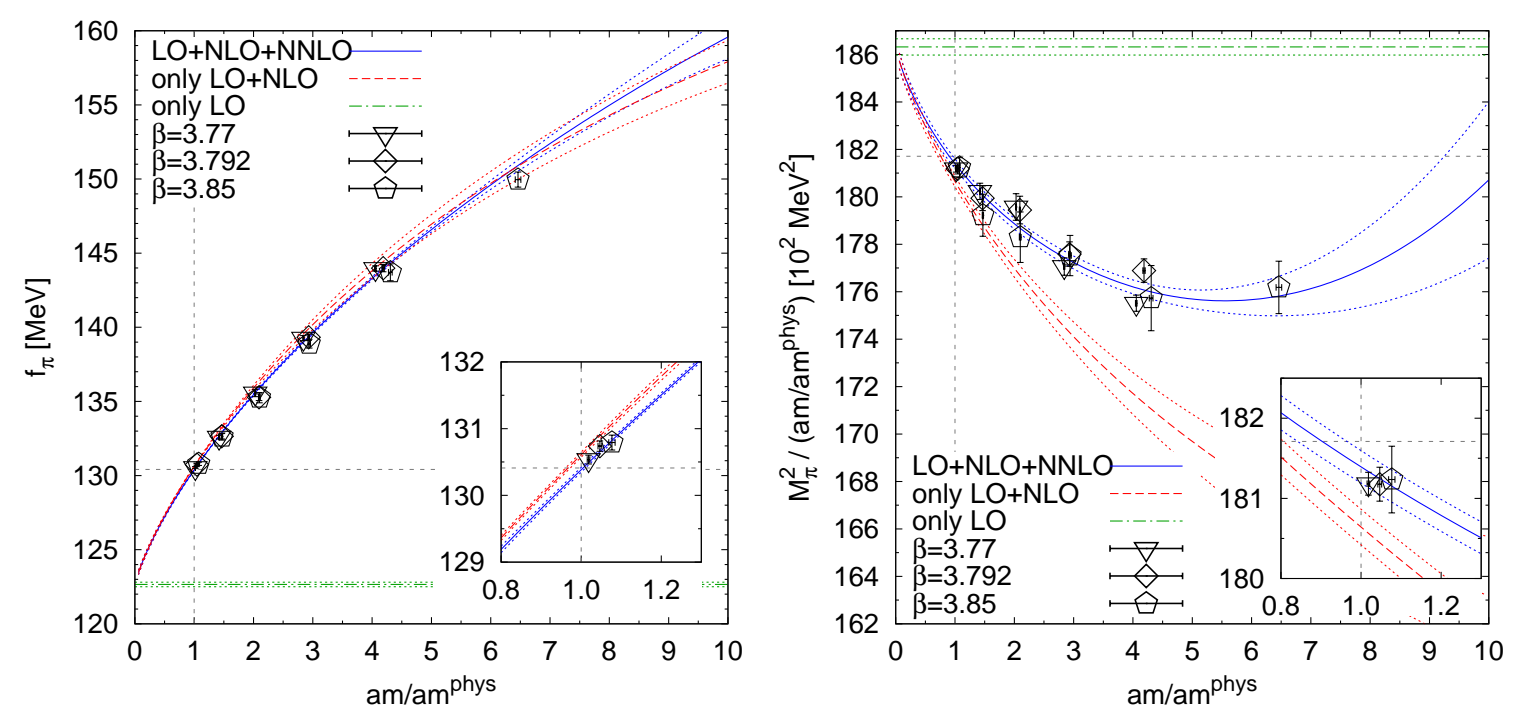

FIG. 20. Combined NNLO fit for lattice scales $1 / a>1.6 \mathrm{GeV}$ and meson masses $135 \mathrm{MeV} \leq$ $M_{\pi} \leq 340 \mathrm{MeV}$ using priors for $\Lambda_{12}, k_{M^{2}}$, and $k_{f}$. Left panel: meson decay constant, right panel: squared meson mass divided by the quark-mass ratio. The solid blue lines show the complete (up to NNLO) fit, whereas the dashed red lines show the LO+NLO contribution of the full NNLO fit, and the dash-dotted green lines show the LO contribution. Only data points included in the fit range are depicted in the plots; the physical values are marked by dashed gray lines.

showing again the stabilizing effect of using the priors.

In Figs.21 and 22 we compare the LECs and the ratio $f_{\pi}^{\text {phys }} / f$ from the NNLO fits to different mass ranges using either no priors or one of our choices for the set of priors (all for ensembles with $1 / a>1.6 \mathrm{GeV}$ ). Our final results with total error bands from the NLO fits are always indicated by the blue lines. In the case of $\bar{\ell}_{3}$, the fits using priors shift the value of the LECs a bit upward, whereas $\bar{\ell}_{4}$ and $f_{\pi}^{\text {phys }} / f$ just fluctuate within the total error band from the NLO fit. The comparison for the LEC $\bar{\ell}_{12}$ again demonstrates the stabilizing effect of the priors or - in other words - the difficulties we encountered in the fits without using priors. In the latter case (top right panel of Fig.22), the fit result only comes close to the phenomenological estimate, Eq. (30), for fit ranges including meson masses of $390 \mathrm{MeV}$ or higher. 

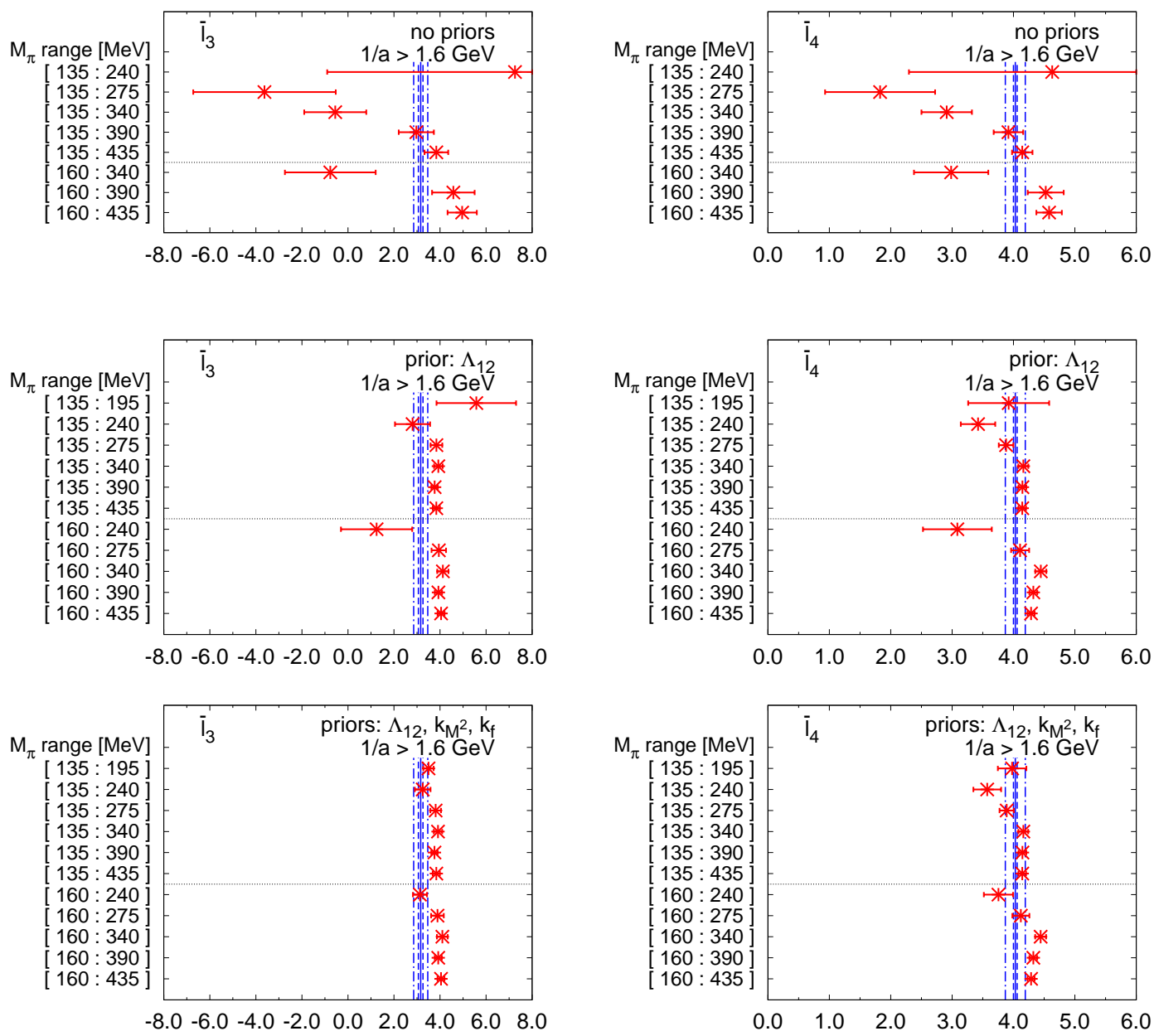

FIG. 21. Results for the LECs $\bar{\ell}_{3}$ (left panels) and $\bar{\ell}_{4}$ (right panels) from NNLO fits to ensembles with $1 / a>1.6 \mathrm{GeV}$ and different mass ranges. The top, middle, and bottom panels show the results using no priors, a prior for $\Lambda_{12}$, and priors for $\Lambda_{12}, k_{M^{2}}$, and $k_{f}$, respectively. The solid, dashed, and dashed dotted blue lines indicate the central value, statistical and total (statistical plus systematic) error bands from our NLO fits, cf. Eqs. (16)-(22).

\section{CONCLUSIONS}

In this paper we presented a determination of the NLO low-energy constants $\bar{\ell}_{3}$ and $\bar{\ell}_{4}$ of $\mathrm{SU}(2)$ chiral perturbation theory from $2+1$ flavor lattice simulations with staggered fermions. In addition we gave results for the LO quantities $f\left(\right.$ or $\left.f_{\pi}^{\text {phys }} / f\right)$ and $B$ (or $\left.\chi^{\text {phys }}=B m^{\text {phys }}\right)$. The quantities $\bar{\ell}_{3,4}$ are also expressed in terms of the scales $\Lambda_{3,4}$, respectively. 

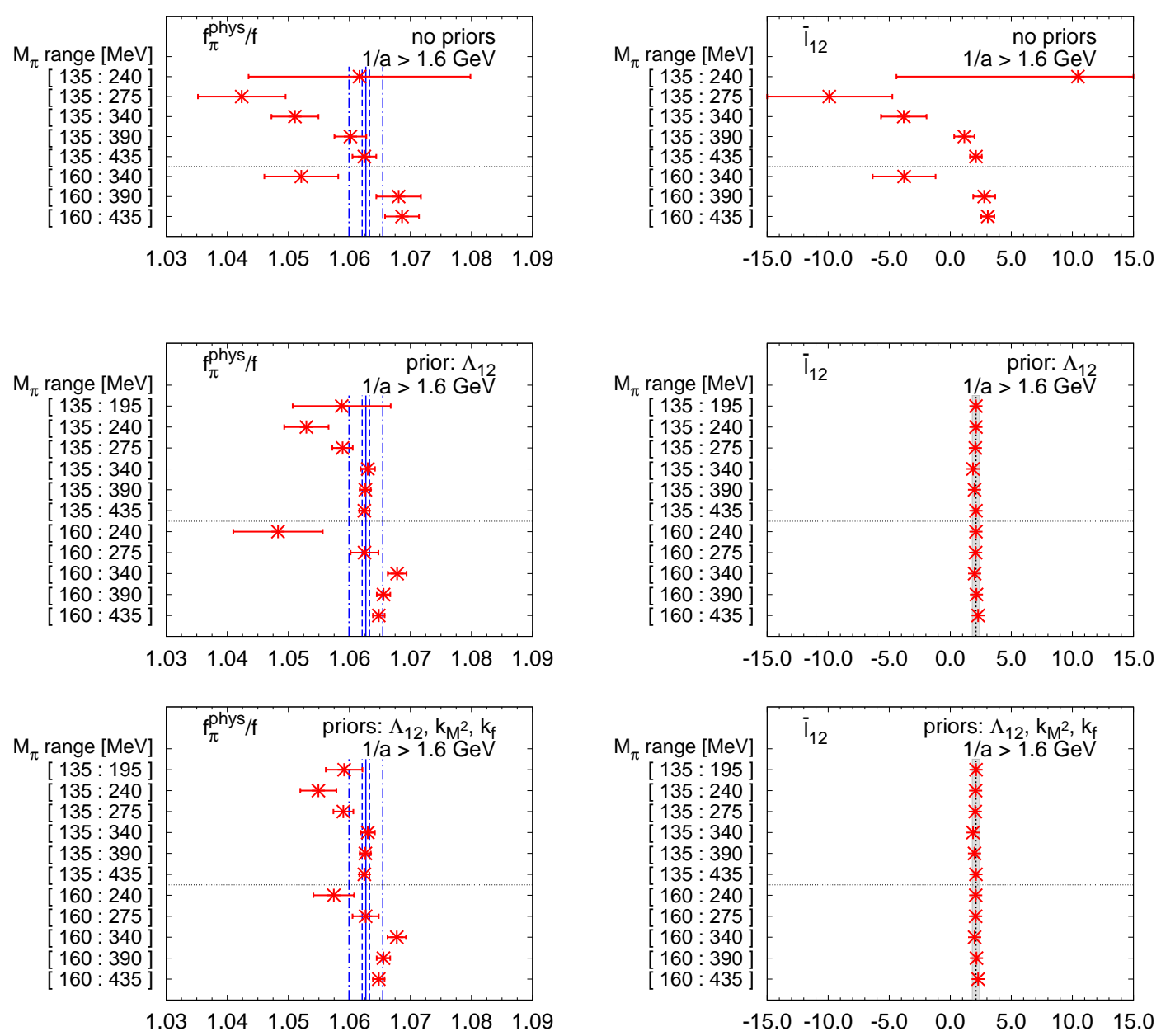

FIG. 22. Results for the ratio $f_{\pi}^{\text {phys }} / f$ (left panels) and the LEC $\bar{\ell}_{12}$ (right panels) from NNLO fits to ensembles with $1 / a>1.6 \mathrm{GeV}$ and different mass ranges. The top, middle, and bottom panels show the results using no priors, a prior for $\Lambda_{12}$, and priors for $\Lambda_{12}, k_{M^{2}}$, and $k_{f}$, respectively. The solid, dashed, and dashed dotted blue lines indicate the central value, statistical and total (statistical plus systematic) error bands from our NLO fits, cf. Eqs. (16)-(22). The gray shaded areas indicate the used prior and its width (where applicable).

Our final results as presented in Eqs. (16)-(22) stem from fits which use the NLO functional form. The result for $\chi^{\text {phys }}$ amounts to a condensate parameter

$$
B(2 \mathrm{GeV})=2.682(36)(39) \mathrm{GeV}
$$

if one divides out the value of the average light quark mass from Refs. [29, 30]. Moreover, 
after multiplying with $F^{2}=f^{2} / 2$ from Eq. (17), one obtains

$$
\Sigma(2 \mathrm{GeV})=2.020(27)(31) 10^{-2} \mathrm{GeV}^{3} \quad \text { or } \quad \Sigma(2 \mathrm{GeV})^{1 / 3}=0.2723(12)(14) \mathrm{GeV}
$$

where all quantities given at the scale $\mu=2 \mathrm{GeV}$ refer to the $\overline{\mathrm{MS}}$ scheme. These results are reasonably consistent with the high-quality entries in Table 10 of Ref. [18], in particular with those by the MILC [17], RBC/UKQCD [31], and ETM [32] collaborations, to mention some of the most precise determinations. Similarly, our results

$$
\bar{\ell}_{3}=3.16(10)(29) \quad \text { and } \quad \bar{\ell}_{4}=4.03(03)(16)
$$

as stated in Eqs. (20) and (21), respectively, are in good agreement with the broad majority of the entries in Table 12 of Ref. [18], in particular with those by the MILC [33], RBC/UKQCD [31], and ETM [32] collaborations. Finally, our result (22) for the ratio $f_{\pi}^{\text {phys }} / f$ agrees with the entries of Table 11 in Ref. [18], perhaps with some slight tension when compared to the recent $2+1+1$ flavor result by the ETM Collaboration [34].

We have carefully examined the effect of various cuts on the data, in particular the effect of requesting $a^{-1}>1.6 \mathrm{GeV}$ and the effect of limiting the pion mass range that enters the chiral fit. It turns out that the restriction to fine lattices improves the quality of the fits, and with this restriction reasonably stable NLO results are obtained for pion mass windows up to $\sim 400 \mathrm{MeV}$. Given the fine grained set of pion masses available in our ensemble basis, we can even explore the effect of dropping some of the lighter data points. We find that $\bar{\ell}_{3}$ is much more robust in this respect than $\bar{\ell}_{4}$ (or $f_{\pi}^{\text {phys }} / f$ ), as is evident from Fig.9.

Finally, we have explored the effect of adding the NNLO contribution to the functional Ansatz. To prevent a dramatic increase in the number of free parameters, we add priors for the new NLO combinations (in which we are not interested) and the genuine NNLO coefficients (to which our data show little sensitivity). We find it very reassuring that these prior-aided NNLO fits remain stable (for a reasonable range of lattice spacings and pion mass windows) and that the resulting fits show a very natural ordering between LO, NLO, and NNLO contributions (out to $M_{\pi} \sim 400 \mathrm{MeV}$ ). Moreover, the NLO coefficients $\bar{\ell}_{3}$ and $\bar{\ell}_{4}$ as determined from these prior-aided NNLO fits are in good agreement with the results of the direct NLO fits. We take this as a sign that our assessment of the systematic uncertainties of these quantities is true and fair.

The chiral fits presented in Secs. [V and $\mathrm{V}$ do not include terms designed to absorb cutoff effects in the data. The purpose of the present work was to explore whether such terms 
are mandatory; we find that they are not (with the level of precision of our data), albeit at the price of pruning the data set to include only lattices with $a^{-1}>1.6 \mathrm{GeV}$. Still, we did perform an exploratory analysis with such terms included, and it seems that with our choice of the scaling trajectory (cf. discussion in the beginning of Sec.IV), there is hardly any change.

In summary, our results (16)-(22), (34), and (35) indicate that $\mathrm{SU}(2)$ chiral low-energy constants can be determined on the lattice with a precision at the level of a few percent for the LO quantities $B, \Sigma, f$, and the level of $O(10 \%)$ for the NLO scales $\Lambda_{3}, \Lambda_{4}$.

\section{ACKNOWLEDGMENTS}

Computations were performed using HPC resources from FZ Jülich and on clusters at Wuppertal University. This work is supported in part by EU Grants PITN-GA-2009-238353 (ITN STRONGnet) and PIRG07-GA-2010-268367 and DFG Grants FO 502/2 and SFBTR 55 .

[1] H. Fritzsch, M. Gell-Mann, and H. Leutwyler, Phys.Lett. B47, 365 (1973).

[2] J. Gasser and H. Leutwyler, Annals Phys. 158, 142 (1984).

[3] J. Gasser and H. Leutwyler, Nucl.Phys. B250, 465 (1985).

[4] K. G. Wilson, Phys.Rev. D10, 2445 (1974).

[5] M. Creutz, Phys.Rev. D21, 2308 (1980).

[6] S. Dürr et al., Science 322, 1224 (2008), arXiv:0906.3599.

[7] E. E. Scholz et al., PoS LATTICE2011, 142 (2011), arXiv:1111.3729.

[8] M. Lüscher and P. Weisz, Phys.Lett. B158, 250 (1985).

[9] C. Morningstar and M. J. Peardon, Phys.Rev. D69, 054501 (2004), arXiv:hep-lat/0311018.

[10] Y. Aoki et al., JHEP 0601, 089 (2006), arXiv:hep-lat/0510084.

[11] S. Dürr et al., Phys.Rev. D79, 014501 (2009), arXiv:0802.2706.

[12] Y. Aoki et al., Phys.Lett. B643, 46 (2006), arXiv:hep-lat/0609068.

[13] Y. Aoki et al., Nature 443, 675 (2006), arXiv:hep-lat/0611014.

[14] Y. Aoki et al., JHEP 0906, 088 (2009), arXiv:0903.4155. 
[15] S. Borsanyi et al., JHEP 1009, 073 (2010), arXiv:1005.3508.

[16] S. Borsanyi et al., JHEP 1011, 077 (2010), arXiv:1007.2580.

[17] A. Bazavov et al., Rev.Mod.Phys. 82, 1349 (2010), arXiv:0903.3598.

[18] G. Colangelo et al., Eur.Phys.J. C71, 1695 (2011), arXiv:1011.4408.

[19] Particle Data Group, K. Nakamura et al., J.Phys.G G37, 075021 (2010).

[20] L. Del Debbio, L. Giusti, M. Lüscher, R. Petronzio, and N. Tantalo, JHEP 0702, 082 (2007), arXiv:hep-lat/0701009.

[21] LHPC Collaboration, J. Bratt et al., Phys.Rev. D82, 094502 (2010), arXiv:1001.3620.

[22] G. Colangelo, S. Dürr, and C. Haefeli, Nucl.Phys. B721, 136 (2005), arXiv:hep-lat/0503014.

[23] C. Aubin and C. Bernard, Phys. Rev. D68, 034014 (2003), arXiv:hep-lat/0304014.

[24] C. Aubin and C. Bernard, Phys. Rev. D68, 074011 (2003), arXiv:hep-lat/0306026.

[25] T. Blum et al., Phys.Rev. D55, 1133 (1997), arXiv:hep-lat/9609036.

[26] MILC Collaboration, K. Orginos, D. Toussaint, and R. Sugar, Phys.Rev. D60, 054503 (1999), arXiv:hep-lat/9903032.

[27] H. Wittig, PoS LATTICE2011, 025 (2012), arXiv:1201.4774.

[28] G. Colangelo, J. Gasser, and H. Leutwyler, Nucl.Phys. B603, 125 (2001), arXiv:hep$\mathrm{ph} / 0103088$.

[29] S. Dürr et al., Phys.Lett. B701, 265 (2011), arXiv:1011.2403.

[30] S. Dürr et al., JHEP 1108, 148 (2011), arXiv:1011.2711.

[31] RBC/UKQCD Collaborations, Y. Aoki et al., Phys.Rev. D83, 074508 (2011), arXiv:1011.0892.

[32] ETM Collaboration, R. Baron et al., JHEP 1008, 097 (2010), arXiv:0911.5061.

[33] A. Bazavov et al., PoS LATTICE2010, 083 (2010), arXiv:1011.1792.

[34] R. Baron et al., JHEP 1006, 111 (2010), arXiv:1004.5284. 